\title{
THE SPACE OF POINT HOMOTOPIC MAPS INTO THE CIRCLE $\left({ }^{1}\right)$
}

\author{
BY \\ B. J. EISENSTADT
}

1. Introduction. The space $C(X)$ of real bounded continuous functions on a topological space has been studied extensively ([9], [7], [6](2), etc.). More recently some of this theory has been extended to the space of functions into certain Banach spaces [5].

In the present paper, we consider the space of point-homotopic continuous maps into the circle. The circle, $R_{2 q}$ (reals $\bmod 2 q$ ), can be made into an abelian group, complete under an invariant metric. Then $R_{2 q}(X)$, the space of point-homotopic continuous functions from $X$ into $R_{2 q}$, is in a natural way an abelian group, complete under an invariant metric. We give a characterization of $R_{2 q}(X)$, for $X$ a compact connected space, as an abelian group, complete under an invariant metric (Theorem 6.4), and a proof that for compact $X$, the metric group properties of $R_{2 q}(X)$ determine the topology on $X$ (Theorem 7.1).

The characterization is obtained by imposing conditions which insure the existence of a pseudo-multiplication by scalars (Theorem 2.2), and the existence of sufficiently many "characters" of the group (Theorems 3.7, 3.10 and 3.11). The points of $X$ are found among the "characters" of the group by investigating certain Banach spaces associated with the group (\$4). Certain new linear functionals are defined and a Banach space characterization of $C(X)$, for compact $X$, is given (Theorem 5.4). That the metric group properties of $R_{2 q}(X)$ determine the topology on a compact $X$ follows quickly from the similar theorem for Banach spaces [10].

2. Some metric group properties of $R_{2 q}(X)$. The circle $R_{2 q}$ is taken to be the factor group of the reals $R$ by the subgroup $I_{2 q}=\{n(2 q)\}$ where $n$ is any integer. Thus $R_{2 q}$ is an abelian group. We denote by $j$ the natural homomorphism of $R$ onto $R_{2 q}$. ( $j_{2 q}$ would be more precise. However, no confusion results from the omission of the subscript.) We define $j^{-1}: R_{2 q} \rightarrow R$ by $j^{-1}(a)=\alpha$ such that $-q<\alpha \leqq q$ and $j(\alpha)=a$. It follows immediately that

$$
j\left(j^{-1}(a)\right)=a
$$

and that for $|\alpha|<q, j^{-1}(j(\alpha))=\alpha$.

Presented to the Society, September 5, 1953; received by the editors October 9, 1953.

(1) This paper is a revised version of the author's doctoral dissertation submitted to the University of Michigan. He wishes to thank Professor Sumner B. Myers for his help and encouragement. A portion of the work was done under Office of Naval Research contract N8-onr71400.

(2) Numbers in brackets refer to the bibliography at the end of this paper. 
If we define $\rho(a)=\left|j^{-1}(a)\right|$, then the function $d(a, b)=\rho(a-b)$ is an invariant metric on $R_{2 q}$ under which $R_{2 q}$ is complete. The space of all continuous functions from a topological space $X$ into $R_{2 q}$, denoted by $R_{2 q}^{X}$, is made into a metric abelian group by defining

$\left(f_{1}+f_{2}\right)(x)=f_{1}(x)+f_{2}(x), \rho(f)=\sup _{x \in X}\{\rho(f(x))\}$, and $d\left(f_{1}, f_{2}\right)=\rho\left(f_{1}-f_{2}\right)$.

The metric is invariant, convergence is uniform convergence, and $R_{2 q}^{X}$ is complete. Now the space of point-homotopic continuous functions from $X$ into $R_{2 q}, R_{2 q}(X)$, is the component of the identity in $R_{2 q}^{X}$ [4]. Thus it is a closed subgroup of $R_{2 q}^{X}$, and it too is an abelian group complete under an invariant metric. Moreover, for each $\epsilon>0, U_{\epsilon}=\left\{f \in R_{2 q}(X) \mid \rho(f)<\epsilon\right\}$ generates $R_{2 q}(X)$. In what follows, the word group will denote an abelian group, complete under an invariant metric, and generated by $U_{\epsilon}=\{a \mid \rho(a)<\epsilon\}$ for each $\epsilon>0$. In addition we assume, with no loss of generality, that $q \geqq 1$.

Definition 2.1. If $\alpha \in R$ and $a \in U_{1} \subset R_{2 q}$, then $\alpha a=j\left(\alpha j^{-1}(a)\right)$.

This pseudo-multiplication by real scalars can be extended to $U_{1} \subset R_{2 q}(X)$ by defining $(\alpha f)(x)=\alpha(f(x))$. Since each operation used in Definition 2.1 is continuous $\left(j^{-1}\right.$ is continuous on $\left.U_{1}\right)$, the function $\alpha f \in R_{2 q}^{X}$. Moreover $\{t f\}$ for $0 \leqq t \leqq \alpha$ is a homotopy from $\theta(x) \equiv \theta\left({ }^{3}\right)=j(0)$ to $\alpha f$ and so $\alpha f \in R_{2 q}(X)$.

Some of the properties of scalar multiplication in a Banach space are preserved by this pseudo-multiplication. Thus it can be readily verified that, for $\alpha, \beta \in R$ and $a, b \in U_{1} \subset R_{2 q}(X)$, the following relations hold.

(P1) $|\alpha| \rho(a)<1 \rightarrow \beta(\alpha a)=(\beta \alpha)(a)$.

(P2) $(\alpha+\beta) a=\alpha a+\beta a$.

(P3) $\rho(a)+\rho(b)<1 \rightarrow \alpha(a+b)=\alpha a+\alpha b$.

(P4) $|\alpha| \rho(a)<1 \rightarrow \rho(\alpha a)=|\alpha| \rho(a)$.

(P5) $1 a=a$.

Definition 2.2. A group $G$ is a pseudo-Banach space if a multiplication by reals can be defined on $U_{1} \subset G$ which satisfies P1-P5.

Thus we have

Theorem 2.1. $R_{2 q}(X)$ is a pseudo-Banach space.

The next theorem shows that the property of being a pseudo-Banach space is a metric group property.

TheOREm 2.2. A group $G$ is a pseudo-Banach space if and only if, for each $a \in U_{1} \subset G$, there exists a unique isomorphic isometry, $i_{a}:[0, \rho(a)] \rightarrow G$ such that $i_{a}(\rho(a))=a .\{[0, \rho(a)]$ represents the closed interval in $R$ with end points at 0 and $\rho(a)$. The isomorphism applies whenever $\alpha, \beta$ and $\alpha+\beta$ all belong to $[0, \rho(a)]$.

Proof. (a) Suppose $G$ is a pseudo-Banach space. For each $a \in U_{1} \subset G$, de-

$\left.{ }^{3}\right)$ The symbol $\theta$ will denote the identity element in a group. The symbol 0 will be reserved for the zero of the reals. 
fine $i_{a}(\alpha)=(\alpha / \rho(a)) a$. Then by $\mathrm{P} 5, i_{a}(\rho(a))=a$; by $\mathrm{P} 2, i_{a}$ is an isomorphism; and by $\mathrm{P} 4, i_{a}$ is an isometry. Now suppose $i_{a}^{\prime}$ is another such map. It follows from P5 and P2 that for $m$ any positive integer $m a=\sum_{t=1}^{m} a$. Thus for $m$ and $n$ positive integers such that $m \leqq n$ we have $i_{a}(m \rho(a) / n)=(m / n) a$ $=(m / n)\left(i_{a}^{\prime}(\rho(a))\right)=(m / n)\left(n i_{a}^{\prime}(\rho(a) / n)\right)$ and $i_{a}^{\prime}(m \rho(a) / n)=m i_{a}^{\prime}(\rho(a) / n)$. But by $\mathrm{P} 1, m i_{a}^{\prime}(\rho(a) / n)=(m / n)\left(n i_{a}^{\prime}(\rho(a) / n)\right)$. Thus $i_{a}$ and $i_{a}^{\prime}$ are equal on a dense set of $[0, \rho(a)]$ and since they are isometries they must be identical $\left(^{4}\right)$.

(b) Suppose $i_{a}$ is a unique isomorphic isometry taking $\rho(a)$ into $a$. For $\alpha \in R$ and $\alpha>0$ define $\bar{\alpha}$ to be the smallest integer such that $\bar{\alpha} \geqq \alpha$. Define

$$
\begin{array}{ll}
\alpha a=\bar{\alpha}\left[i_{a}\left(\frac{\alpha}{\bar{\alpha}} \rho(a)\right)\right] & \text { for } \alpha>0, \\
\alpha a=\theta & \text { for } \alpha=0, \\
\alpha a=-((-\alpha) a) & \text { for } \alpha<0 .
\end{array}
$$

Since inverses and multiplication by integers are well defined in any group, the preceding definitions give a precise meaning to $\alpha a$.

The proof that this multiplication satisfies P1-P5 involves much intricate detail and is not given here. It may be found in the author's dissertation.

LemMA 2.1. If $G$ is a pseudo-Banach space, then for each $b \in G$ and each $\epsilon$ such that $0<\epsilon \leqq 1$, there exists $\alpha \in R$ and $a \in U_{\epsilon}$ such that $\alpha a=b$.

Proof. Since $U_{\epsilon}$ generates $G$, there exist elements $a_{1}, \cdots, a_{n}$ in $U_{\epsilon}$ such that $b=\sum_{i=1}^{n} a_{i}$. Choose $\alpha \geqq n$ and let $a=\sum_{i=1}^{n}(1 / \alpha) a_{i}$. Then

$$
\rho(a) \leqq \sum_{i=1}^{n} \rho\left((1 / \alpha) a_{i}\right) .
$$

By P4, $\rho\left((1 / \alpha) a_{i}\right)=(1 / \alpha) \rho\left(a_{i}\right)$ and so

$$
\rho(a) \leqq \sum_{i=1}^{n} \rho\left(\frac{1}{\alpha} a_{i}\right)=\sum_{i=1}^{n} \frac{1}{\alpha} \rho\left(a_{i}\right)<\frac{n \epsilon}{\alpha} \leqq \epsilon .
$$

Thus $a \in U_{\epsilon}$. Moreover $\sum_{i=1}^{n} \rho\left((1 / \alpha) a_{i}\right)<\epsilon \leqq 1$ and so by repeated application of $\mathrm{P} 3 \alpha a=\sum_{i=1}^{n} \alpha\left((1 / \alpha) a_{i}\right)$. But by P1, $\alpha\left((1 / \alpha) a_{i}\right)=a_{i}$ and so $\alpha a=\sum_{i=1}^{n} a_{i}$ $=b$.

LEммA 2.2. If $G$ a is pseudo-Banach space, and if for $a \in U_{1}$ and $\alpha \in R$ and different from zero, $\alpha a=\theta$, then either $a=\theta$ or $\rho(a) \geqq 2 /|\alpha|$.

Proof. If $\theta=\alpha a=(\alpha / 2+\alpha / 2) a$ then, by P2, $(\alpha / 2) a=-((\alpha / 2) a)=(-\alpha / 2) a$ and $(1 / 2)((\alpha / 2) a)=(1 / 2)((-(\alpha / 2)) a)$. Now if $\rho(a)<2 /|\alpha|$, then $|\alpha / 2| \rho(a)$ $<1)$, and we have by $\mathrm{P} 1$ that $(1 / 2)((\alpha / 2) a)=(\alpha / 4) a$ and $(1 / 2)((-\alpha / 2) a)$

(4) Since P3 was not used in establishing the existence and uniqueness of $i_{a}$, the proof of sufficiency will prove that $\mathrm{P} 3$ is a consequence of P1, P2, P4, and P5. This can easily be established directly. 
$=(-\alpha / 4) a$ and $(\alpha / 4) a=(-\alpha / 4) a=-(\alpha / 4) a$. Thus $\theta=(\alpha / 4) a+(\alpha / 4) a$ $=(\alpha / 2) a$. But by P4, $\rho((\alpha / 2) a)=|\alpha / 2| \rho(a)$ and if this is zero, $\rho(a)=0$ and $a=\theta$.

Lemma 2.3. If $G$ a pseudo-Banach space, the map of $R \times U_{1} \rightarrow G$ given by $(\alpha, a) \rightarrow \alpha a$ is continuous.

Proof. We show that the neighborhood $V$ of $\left(\alpha_{0}, a_{0}\right)$ defined by $V$ $=\left\{(\alpha, a)|| \alpha-\alpha_{0} \mid<\min [\epsilon / 2,1 / \rho(a)]\right.$ and $\rho\left(a-a_{0}\right)<\min \left[\epsilon / 2\left|\alpha_{0}\right|, 1 /\left|\alpha_{0}\right|\right.$, $\left.\left.1-\rho\left(a_{0}\right)\right]\right\}$ maps into the $\epsilon$ neighborhood of $\alpha_{0} a_{0}$.

For $(\alpha, a) \in V$

$$
\begin{aligned}
\alpha a-\alpha_{0} a_{0} & =\alpha\left(a-a_{0}\right)+\alpha a_{0}-\alpha_{0} a_{0}=\alpha\left(a-a_{0}\right)+\left(\alpha-\alpha_{0}\right) a_{0} \\
& =\alpha_{0}\left(a-a_{0}\right)+\left(\alpha-\alpha_{0}\right)\left(a-a_{0}\right)+\left(\alpha-\alpha_{0}\right) a_{0} \\
& =\alpha_{0}\left(a-a_{0}\right)+\left(\alpha-\alpha_{0}\right) a
\end{aligned}
$$

by $\mathrm{P} 3, \mathrm{P} 2, \mathrm{P} 2$, and $\mathrm{P} 3$ respectively. Then $\rho\left(\alpha a-\alpha_{0} a_{0}\right) \leqq \rho\left(\alpha_{0}\left(a-a_{0}\right)\right)$ $+\rho\left(\left(\alpha-\alpha_{0}\right) a\right)<\epsilon / 2+\epsilon / 2<\epsilon$ by $\mathrm{P} 1$ and $\mathrm{P} 4$.

In what follows we shall use properties P1-P5 without specific reference, taking care always that the hypotheses of the statements are satisfied.

Definition 2.3. An element $h \in G$ is a root of unity if there exists an integer $n$ such that $n h=\theta$. The set of roots of unity we denote by $H$ and the closure of $H$ in $G$ by $\bar{H}$.

$H$ and $\bar{H}$ are subgroups of $G$ in the usual sense.

The elements of $\bar{H} \subset R_{2 q}(X)$ have special metric properties as well. The following lemma makes this explicit for the case where $X$ is a connected space.

Lemma 2.4. If $X$ is connected, then $h \in \bar{H} \subset R_{2 q}(X)$ is a constant function and if $\rho(h)<1$, then for $g \in R_{2 q}(X)$ such that $\rho(h)+\rho(g)<1$ we have either $\rho(h+g)$ $=\rho(h)+\rho(g)$ or $\rho(h-g)=\rho(h)+\rho(g)$.

Proof. Suppose $h \in H \subset R_{2 q}(X)$. Then there exists an integer $n$ such that $n(h(x)) \equiv \theta$ for all $x \in X$. Thus $h(X) \subset A=\left\{a \in R_{2 q} \mid n a=\theta\right\}$. But $h(X)$ is connected while the set $A$ is discrete and so $h$ is a constant function. The definition of the metric then implies that the elements of $\bar{H}$ are constant functions. From this fact plus the definition of the function $\rho$, the second part follows immediately.

We are led to the following definitions.

Definition 2.4. If $\rho(a)+\rho(b)<1$ and if $\rho(a)+\rho(b)=\rho(a+b)$, then the pair $\{a, b\}$ is positive.

Definition 2.5. If $a \in U_{1} \subset G$ and if for all $b \in U_{1-\rho(a)} \subset G$ either $\{a, b\}$ or $\{a,-b\}$ is positive, then $a$ is a constant of $G$.

Definition 2.6. A pseudo-Banach space $G$ is a space with constants if $\bar{H} \neq\{\theta\}\left(^{5}\right)$ and if all the elements of $\bar{H} \cap U_{1}$ are constants of $G$.

(5) We assume $\bar{H} \neq\{\theta\}$, as otherwise $G$ is essentially a Banach space. 
TheOREM 2.3. If $X$ is a connected space, then $R_{2 q}(X)$ is a space with constants.

Proof. Theorem 2.1 and Lemma 2.4 .

3. Subspaces and characters. A basic theorem in the classification of Banach spaces is that every Banach space $B$ is equivalent to a closed subspace of $C(X)$ for some compact topological space $X$ [1]. The points of $X$ are found among the continuous linear functionals on $B$. The existence of suffciently many such functionals is assured by the Hahn-Banach theorem [ 3 , p. 55]. In this section we prove that under modified definitions of equivalence and subspace, every space with constants is equivalent to a subspace of $R_{2 q}(X)$ for some $q \geqq 1$ and for some compact connected space $X$.

Definition 3.1. Two groups $G$ and $\widehat{G}$ are equivalent if there is an isomorphism $I: G \rightarrow \widehat{G}$ such that $I$ is an isometry on $U_{1}$ and such that $I\left(U_{1}\right)=\widehat{O}_{1}$. \{For this definition we do not require that $G$ and $\widehat{G}$ be complete. It is clear that the relation of equivalence is symmetric, reflexive, and transitive.

Definition 3.2. A subset $G^{\prime}$ of a pseudo-Banach space $G$ is a subspace of $G$ if $G^{\prime}$ is a subgroup (in the ordinary sense) and if, for $\alpha \neq 0$ and $a \in U_{1} \subset G$, $\alpha a \in G^{\prime}$ if and only if $a \in G^{\prime}$.

Definition 3.3. If $G^{\prime}$ is a subspace of a pseudo-Banach space $G$, then $L: G^{\prime} \rightarrow R_{2 q}$ is a character of $G^{\prime}$ if

( $\left.\mathrm{P}^{\prime} 1\right) L(a+b)=L(a)+L(b)$,

$\left(\mathrm{P}^{\prime} 2\right)\left|j^{-1}(L(a))\right| \leqq \rho(a)$ whenever $\rho(a)<1$,

$\left(\mathrm{P}^{\prime} 3\right) \quad L(\alpha a)=\alpha L(a)$ whenever $\rho(a)<1$.

From the definitions it is clear that $G^{\prime}$ may be all of $G$.

THEOREM 3.1. The characters of a subspace $G^{\prime}$ of a pseudo-Banach space $G$ are continuous on $G^{\prime}$.

Proof. By $\mathrm{P}^{\prime} 1, L$ is a homomorphism. But for $0<\epsilon<1$ and $a \in U_{\epsilon} \cap G^{\prime}$,

$$
\left|j^{-1}(L(a))\right|<\epsilon
$$

and so $L$ is continuous at the identity and therefore continuous on $G^{\prime}$.

THEOREM 3.2. If $G^{\prime}$ is a subspace of a pseudo-Banach space $G$ and if $L: G^{\prime}$ $\rightarrow R_{2 q}$ satisfies $\mathrm{P}^{\prime} 1$ and $\mathrm{P}^{\prime} 2$, then $L$ is a character of $G^{\prime}$.

Proof. For $a \in U_{1} \cap G^{\prime}$ and $n$ any positive integer, $n((1 / n) a)=a$. Then for $m$ any integer $\mathrm{P}^{\prime} 1$ gives $(m / n) L(a)=(m / n) L(n((1 / n) a))=(m / n)(n L((1 / n) a))$. But $R_{2 q}$ is itself a pseudo-Banach space and by $\mathrm{P}^{\prime} 2 n \rho(L((1 / n) a))$ $=n\left|j^{-1}(L((1 / n) a))\right| \leqq n \rho((1 / n) a)=\rho(a)<1$. Thus we have $(m / n)(n L((1 / n) a))$ $=m L((1 / n) a)=L((m / n) a)$ by $\mathrm{P}^{\prime} 1$, and $(m / n) L(a)=L((m / n) a)$. By Theorem 3.1 and Lemma 2.3 both $\alpha L(a)$ and $L(\alpha a)$ are continuous in $\alpha$. Since they are equal on a dense set of $R$, they are equal for all $\alpha \in R$ and $L$ satisfies $\mathrm{P}^{\prime} 3$ on $G^{\prime}$.

The usual boundedness restriction for linear functionals on a Banach 
space would translate here to $\left|j^{-1}(L(a))\right| \leqq M \rho(a)$. However, this plus $\mathrm{P}^{\prime} 1$ does not imply $\mathrm{P}^{\prime} 3$. The proof uses strongly that $M=1$ and the theorem is false without it. For let $G^{\prime} \subset R_{2}([0,1])$ be the set $\left\{f \in R_{2}([0,1]) \mid f(x)=j(\alpha+\beta x)\right\}$ and define $L(j(\alpha+\beta x))=j(\alpha-(3 / 2) \beta)$. It is easily verified that $L$ satisfies $\mathrm{P}^{\prime} 1$ and that $\left|j^{-1} L(f)\right| \leqq 4 \rho(f)$. However,

$$
(1 / 2) L(j(-1 / 2+x))=(1 / 2) j(-1 / 2-3 / 2)=(1 / 2) j(-2)=(1 / 2) \theta=\theta,
$$

while $\left.L((1 / 2) j(-1 / 2+x))=L\left(j(1 / 2) j^{-1} j(-1 / 2+x)\right)\right)=L(j(-1 / 4+(1 / 2) x))$ $=j(-1 / 4-3 / 4)=j(-1) \neq \theta$.

Since in the construction of characters we have no other way of insuring that $\mathrm{P}^{\prime} 3$ be satisfied we must use the stronger form given by $\mathrm{P}^{\prime} 2$.

TheOREM 3.3. If $G^{\prime}$ is a subspace of a pseudo-Banach space $G$ and $L^{\prime}$ is a character of $G^{\prime}$, then there exists a character $L$ of $G$ such that $L=L^{\prime}$ on $G^{\prime}$.

Proof. The proof is a modification of the similar theorem for Banach spaces [3, p. 28]. If $G^{\prime}=G$ we are through. If $G^{\prime} \neq G$, there exists an element $a \in\left(G-G^{\prime}\right) \cap U_{1 / 2}$, since $U_{1 / 2}$ generates $G$. For $b_{1}$ and $b_{2}$ any elements of $G^{\prime} \cap U_{1 / 2}$ and for $\beta_{1}$ and $\beta_{2}$ real numbers such that $0<\beta_{i} \leqq 1$ and $\beta=\min \left(\beta_{1}, \beta_{2}\right)$ we have, by $\mathrm{P}^{\prime} 1$ and $\mathrm{P}^{\prime} 2$, that

$$
\begin{aligned}
j^{-1}\left\{L^{\prime}\left(\frac{\beta}{\beta_{1}} b_{1}\right)-L^{\prime}\left(\frac{\beta}{\beta_{2}} b_{2}\right)\right\} & =j^{-1}\left\{L^{\prime}\left(\frac{\beta}{\beta_{1}} b_{1}-\frac{\beta}{\beta_{2}} b_{2}\right)\right\} \\
& \leqq \rho\left(\frac{\beta}{\beta_{1}} b_{1}-\frac{\beta}{\beta_{2}} b_{2}\right)
\end{aligned}
$$

since

$$
\begin{gathered}
\left|j^{-1}\left(L^{\prime}\left(\frac{\beta}{\beta_{1}} b_{1}\right)\right)\right|+\left|j^{-1}\left(L^{\prime}\left(\frac{\beta}{\beta_{2}} b_{2}\right)\right)\right|<1 \\
j^{-1}\left\{L^{\prime}\left(\frac{\beta}{\beta_{1}} b_{1}\right)-L^{\prime}\left(\frac{\beta}{\beta_{2}} b_{2}\right)\right\}=j^{-1}\left\{L^{\prime}\left(\frac{\beta}{\beta_{1}} b_{1}\right)\right\}-j^{-1}\left\{L^{\prime}\left(\frac{\beta}{\beta_{2}} b_{2}\right)\right\}
\end{gathered}
$$

and so

$$
\begin{aligned}
j^{-1}\left\{L^{\prime}\left(\frac{\beta}{\beta_{1}} b_{1}\right)\right\}-j^{-1}\left\{L^{\prime}\left(\frac{\beta}{\beta_{2}} b_{2}\right)\right\} & \leqq \rho\left(\frac{\beta}{\beta_{1}} b_{1}-\frac{\beta}{\beta_{2}} b_{2}\right) \\
& \leqq \rho\left(\frac{\beta}{\beta_{1}} b_{1}+\beta a\right)+\rho\left(\frac{\beta}{\beta_{2}} b_{2}+\beta a\right)
\end{aligned}
$$

and so

$$
-\frac{\beta}{\beta_{2}} \rho\left(b_{2}+\beta_{2} a\right)-\frac{\beta}{\beta_{2}} j^{-1}\left(L^{\prime}\left(b_{2}\right)\right) \leqq \frac{\beta}{\beta_{1}} \rho\left(b_{1}+\beta_{1} a\right)-\frac{\beta}{\beta_{1}} j^{-1}\left(L^{\prime}\left(b_{1}\right)\right) .
$$


Dividing by $\beta$ gives

$$
-\frac{1}{\beta_{2}} \rho\left(b_{2}+\beta_{2} a\right)-\frac{1}{\beta_{2}} j^{-1}\left(L^{\prime}\left(b_{2}\right)\right) \leqq \frac{1}{\beta_{1}} \rho\left(b_{1}+\beta_{1} a\right)-\frac{1}{\beta_{1}} j^{-1}\left(L^{\prime}\left(b_{1}\right)\right) .
$$

Since (3.1) holds for all $\beta_{1}, \beta_{2}, b_{1}$, and $b_{2}$ we have

$$
\begin{aligned}
m & =\underset{b_{2}, \beta_{2}}{\text { l.u.b. }}\left\{-\frac{1}{\beta_{2}} \rho\left(b_{2}+\beta_{2} a\right)-\frac{1}{\beta_{2}} j^{-1}\left(L^{\prime}\left(b_{2}\right)\right)\right\} \\
& \leqq \underset{\text { g.l.b. }}{b_{1}, \beta_{1}}\left\{\frac{1}{\beta_{1}} \rho\left(b_{1}+\beta_{1} a\right)-\frac{1}{\beta_{1}} j^{-1}\left(L^{\prime}\left(b_{1}\right)\right)\right\}=M .
\end{aligned}
$$

Let $G^{\prime \prime}=\left\{c \in G \mid c=\gamma a+b\right.$ for any $\gamma \in R$ and $\left.b \in G^{\prime}\right\}$. For a fixed $c \in G^{\prime \prime}, \gamma$ and $b$ are uniquely determined. If $\gamma a+b=\gamma^{\prime} a+b^{\prime}$, then $\left(\gamma-\gamma^{\prime}\right) a=b^{\prime}-b \in G^{\prime}$. But $a \notin G^{\prime}$ and $G^{\prime}$ is a subspace, thus $\gamma=\gamma^{\prime}$ and so $b=b^{\prime}$.

Choose $\alpha \in R$ such that $m \leqq \alpha \leqq M$ and define $L^{\prime \prime}: G^{\prime \prime} \rightarrow R_{2 q}$ by $L^{\prime \prime}(c)$ $=j(\gamma \alpha)+L^{\prime}(b)$.

We show that $G^{\prime \prime}$ is a subspace and that $L^{\prime \prime}$ is a character of $G^{\prime \prime}$. That $G^{\prime \prime}$ properly contains $G^{\prime}$ and that $L^{\prime \prime}=L^{\prime}$ on $G^{\prime}$ is immediate.

$G^{\prime \prime}$ is clearly a subgroup (in the usual sense). Suppose $c \in G^{\prime \prime} \cap U_{1}$ and $0 \neq \delta \in R$. Let $\eta=2 \max (|\delta|,(1 / 2)|\delta \gamma|)$. Then $(\eta / \delta)((\delta / \eta) c-(\delta \gamma / \eta) a)$ $=c-\gamma a=b \in G^{\prime}$. Therefore $(\delta / \eta) c-(\delta \gamma / \eta) a \in G^{\prime}$ and $\eta((\delta / \eta) c-(\delta \gamma / \eta) a)$ $=\delta c-(\delta \gamma) a \in G^{\prime}$, and so $\delta c \in G^{\prime \prime}$. Now suppose $\rho(c)<1,0 \neq \delta \in R$, and that $\delta c \in G^{\prime \prime}$. Then $\delta c=\gamma^{\prime} a+b^{\prime}$. Let $\eta=\max \left(2,\left|\gamma^{\prime} / \delta\right|\right)$. Then $\eta \delta\left((1 / \eta) c-\left(\gamma^{\prime} / \eta \delta\right) a\right)$ $=b^{\prime} \in G^{\prime}$ and $(1 / \eta) c-\left(\gamma^{\prime} / \eta \delta\right) a \in G^{\prime}$, so that

$$
\eta\left((1 / \eta) c-\left(\gamma^{\prime} / \eta \delta\right) a\right)=c-\left(\gamma^{\prime} / \delta\right) a \in G^{\prime}, \text { and } c \in G^{\prime \prime} .
$$

Thus we have proved that $G^{\prime \prime}$ is a subspace.

Now $L^{\prime \prime}\left(c_{1}+c_{2}\right)=L^{\prime \prime}\left(\gamma_{1} a+b_{1}+\gamma_{2} a+b_{2}\right)=L^{\prime \prime}\left(\left(\gamma_{1}+\gamma_{2}\right) a+\left(b_{1}+b_{2}\right)\right)$ $=j\left(\left(\gamma_{1}+\gamma_{2}\right) \alpha\right)+L^{\prime}\left(b_{1}+b_{2}\right)=j\left(\gamma_{1} \alpha\right)+L^{\prime}\left(b_{1}\right)+j\left(\gamma_{2} \alpha\right)+L^{\prime}\left(b_{2}\right)=L^{\prime \prime}\left(c_{1}\right)+L^{\prime \prime}\left(c_{2}\right)$ and $\mathrm{P}^{\prime} 1$ is satisfied. Now suppose $c=\gamma a+b \in G^{\prime \prime}$ and $\rho(c)<1$. If $\gamma=0, \mathrm{P}^{\prime} 2$ is immediate. If $\gamma \neq 0$, let $\delta=\max (2,|4 / \gamma|)$. Then $\delta \gamma\{(1 / \delta \gamma) c-(1 / \delta) a\}$ $=c-\gamma a=b \in G^{\prime}$ and so $(1 / \delta \gamma) c-(1 / \delta) a \in G^{\prime}$. Moreover $\rho((1 / \delta \gamma) c-(1 / \delta) a)$ $<1 / 4+1 / 4=1 / 2$. Thus in (3.1) we may put $b_{1}=b_{2}=(1 / \delta \gamma) c-(1 / \delta) a$ and $\beta_{1}=\beta_{2}=(1 / \delta)$. We get

$$
\begin{aligned}
-\delta \rho\left(\frac{1}{\delta \gamma} c\right)-\delta\left\{j^{-1}\left(L^{\prime}\left(\frac{1}{\delta \gamma} c-\frac{1}{\delta} a\right)\right)\right\} & \leqq m \leqq \alpha \leqq M \\
& \leqq \delta \rho\left(\frac{1}{\delta \gamma} c\right)-\delta\left\{j^{-1}\left(L^{\prime}\left(\frac{1}{\delta \gamma} c-\frac{1}{\delta} a\right)\right)\right\}
\end{aligned}
$$

and so $\left|\alpha / \delta+j^{-1}\left(L^{\prime}((1 / \delta \gamma) c-(1 / \delta) a)\right)\right| \leqq \rho((1 / \delta \gamma) c)=(1 /|\delta \gamma|) \rho(c)$ and $\mid \gamma \alpha+$ $\delta \gamma j^{-1}\left(L^{\prime}((1 / \delta \gamma) c-(1 / \delta) a)\right) \mid \leqq \rho(c)<1$. But $j^{-1}(j(\beta))=\beta$ for $|\beta| \leqq 1$ and so 


$$
\begin{aligned}
\rho(c) & \geqq\left|j^{-1}\left\{j(\gamma \alpha)+j\left(\delta \gamma j^{-1}\left(L^{\prime}\left(\frac{1}{\delta \gamma} c-\frac{1}{\delta} a\right)\right)\right)\right\}\right| \\
& =\left|j^{-1}\left\{j(\gamma \alpha)+\delta \gamma\left(L^{\prime}\left(\frac{1}{\delta \gamma} c-\frac{1}{\delta} a\right)\right)\right\}\right| \\
& =\left|j^{-1}\left\{j(\gamma \alpha)+L^{\prime}\left(\delta \gamma\left(\frac{1}{\delta \gamma} c-\frac{1}{\delta} a\right)\right)\right\}\right|
\end{aligned}
$$

as $L^{\prime}$ is a character on $G^{\prime}$ and satisfies $\mathrm{P}^{\prime} 3$. Thus $\rho(c) \geqq\left|j^{-1}\left\{j(\gamma \alpha)+L^{\prime}(b)\right\}\right|$ $=\left|j^{-1}\left\{L^{\prime \prime}(c)\right\}\right|$ and $L^{\prime \prime}$ satisfies $\mathrm{P}^{\prime} 2$ on $G^{\prime \prime}$.

By Theorem 3.2, $L^{\prime \prime}$ is a character of $G^{\prime \prime}$. Then by transfinite induction there exists a character $L$ of $G$ such that $L=L^{\prime}$ on $G^{\prime}$.

Theorem 3.3 does not prove the existence of characters on a pseudoBanach space $G$. We must first exhibit a subspace $G^{\prime}$ of $G$ and a character of $G^{\prime}$. At first glance, the real multiples of an element in $U_{1}$ might seem to do for $G^{\prime}$. But this is not necessarily a subspace of $G$ (Corollary 1 to Theorem 3.5). We show, however, that $\bar{H}$ is a subspace of $G$ and that if $G$ is a space with constants, there exists a character taking $\bar{H}$ into $R_{2 q}$ for some $q \geqq 1$.

TheOREM 3.4. If $G$ is a pseudo-Banach space, $\bar{H}$ is a subspace of $G$.

Proof. Suppose $h \in \bar{H} \cap U_{1}$ and $0 \neq \alpha \in R$. Then there exist $h_{i} \in H \cap U_{1}$ and integers $p_{i}$ and $q_{i}$ such that $h_{i} \rightarrow h$ and $p_{i} / q_{i} \rightarrow \alpha$. Since $h_{i} \in H$, there exist integers $n_{i}$ such that $n_{i} h_{i}=\theta$. Then $n_{i} q_{i}\left(\left(p_{i} / q_{i}\right) h_{i}\right)=p_{i}\left(n_{i} h_{i}\right)=\theta$ and so $\left(p_{i} / q_{i}\right) h_{i} \in H$. But by Lemma 2.3, $\left(p_{i} / q_{i}\right) h_{i} \rightarrow \alpha h$ and so $\alpha h \in \bar{H}$.

Now suppose $0 \neq \alpha \in R, h \in U_{1}$, and $\alpha h \in \bar{H}$. If $\alpha<0$, then $\alpha h=-\{(-\alpha) h\}$ and $(-\alpha) h \in \bar{H}$. Thus we may assume $\alpha>0$. There exist $h_{i} \in H$ such that $h_{i} \rightarrow \alpha h$. Thus there exists an $I$, such that $\rho\left(\alpha h-h_{i}\right)<1$ whenever $i \geqq I$ and so $(1 / \bar{\alpha})\left(\alpha h-h_{i}\right)$ is defined for $i \geqq I$. Moreover $\bar{\alpha}\left[(\alpha / \bar{\alpha}) h-(1 / \bar{\alpha})\left(\alpha h-h_{i}\right)\right]=\alpha h-\alpha h$ $+h_{i} \in H$ and since $\bar{\alpha}$ is an integer, $a_{i}=(\alpha / \bar{\alpha}) h-(1 / \bar{\alpha})\left(\alpha h-h_{i}\right) \in H$. But $a_{i} \rightarrow(\alpha / \bar{\alpha}) h$ and so $(\alpha / \bar{\alpha}) h \in \bar{H}$. Since $(\alpha / \bar{\alpha}) h \in U_{1}$, by the first part of the proof $(\bar{\alpha} / \alpha)((\alpha / \bar{\alpha}) h)=h \in \bar{H}$.

Lemma 3.1. If $\{a, b\}$ is positive (Definition 2.4) and if $\alpha \geqq 0, \beta \geqq 0$, and $\alpha \rho(a)+\beta \rho(b)<1$, then $\{\alpha a, \beta b\}$ is positive.

Proof. For either $\alpha$ or $\beta$ equal to zero, the result is immediate. We assume $\alpha \geqq \beta>0$. Since $\alpha \rho(a)+\beta \rho(b)<1, \quad \alpha \rho(a)=\rho(\alpha a)$ and $\beta \rho(b)=\rho(\beta b)$. Thus $(1 / \alpha) \rho(\alpha a+\beta b) \leqq(1 / \alpha)(\rho(\alpha a)+\rho(\beta b))=\rho(a)+(\beta / \alpha) \rho(b) \leqq \rho(a)+\rho(b)<1$, and $(1 / \alpha) \rho(\alpha a+\beta b)=\rho((1 / \alpha)(\alpha a+\beta b))$. But $\rho(\alpha a)+\rho(\beta b)<1$ and so $(1 / \alpha)(\alpha a+\beta b)$ $=(1 / \alpha)(\alpha a)+(1 / \alpha)(\beta b)$ and since $\alpha \rho(a)<1$ and $\beta \rho(b)<1,(1 / \alpha)(\alpha a+\beta b)$ $=a+(\beta / \alpha) b$. Now $\rho(a+(\beta / \alpha) b)=\rho(a+b-(1-\beta / \alpha) b) \geqq \rho(a+b)-(1-\beta / \alpha) \rho(b)$ $=\rho(a)+\rho(b)-(1-\beta / \alpha) \rho(b)=\rho(a)+(\beta / \alpha) \rho(b)$. But the opposite inequality is always true and so $\rho(a)+(\beta / \alpha) \rho(b)=\rho(a+(\beta / \alpha) b)=\rho((1 / \alpha)(\alpha a+\beta b))$ $=(1 / \alpha) \rho(\alpha a+\beta b)$. Thus $\rho(\alpha a+\beta b)=\alpha \rho(a)+\beta \rho(b)=\rho(\alpha a)+\rho(\beta b)<1$ and so 
$\{\alpha a, \beta b\}$ is positive.

LEммA 3.2. If $G$ is a space with constants, $h_{1} \in \bar{H}, h_{2} \in \bar{H}$, and $\left\{h_{1}, h_{2}\right\}$ is positive, then $\rho\left(h_{1}-h_{2}\right)=\left|\rho\left(h_{1}\right)-\rho\left(h_{2}\right)\right|$.

Proof. $(1 / 2) h_{1}+(1 / 2) h_{2} \in \bar{H} \cap U_{1 / 2}$ and is therefore a constant of $G$. Moreover $\rho\left((1 / 2) h_{1}+(1 / 2) h_{2}\right)+\rho\left((1 / 2) h_{1}-(1 / 2) h_{2}\right) \leqq \rho\left(h_{1}\right)+\rho\left(h_{2}\right)<1$, and so either $\left\{(1 / 2) h_{1}+(1 / 2) h_{2},(1 / 2) h_{1}-(1 / 2) h_{2}\right\}$ or $\left\{(1 / 2) h_{1}+(1 / 2) h_{2},(1 / 2) h_{2}-(1 / 2) h_{1}\right\}$ is positive. If the first pair is positive we have

$$
\begin{aligned}
\rho\left((1 / 2) h_{1}+(1 / 2) h_{2}\right)+\rho & \left((1 / 2) h_{1}-(1 / 2) h_{2}\right) \\
& =\rho\left((1 / 2) h_{1}+(1 / 2) h_{2}+(1 / 2) h_{1}-(1 / 2) h_{2}\right)=\rho\left(h_{1}\right)
\end{aligned}
$$

Thus $\rho\left((1 / 2) h_{1}-(1 / 2) h_{2}\right)=\rho\left(h_{1}\right)-\rho\left((1 / 2) h_{1}+(1 / 2) h_{2}\right)=(1 / 2) \rho\left(h_{1}\right)-(1 / 2)$ $\cdot \rho\left(h_{2}\right)$ by Lemma 3.1. If the second pair is positive we have $\rho\left((1 / 2) h_{1}-(1 / 2) h_{2}\right)$ $=(1 / 2) \rho\left(h_{2}\right)-(1 / 2) \rho\left(h_{1}\right)$. Since $\rho\left((1 / 2) h_{1}-(1 / 2) h_{2}\right) \geqq 0$ we have in either case that $\rho\left((1 / 2) h_{1}-(1 / 2) h_{2}\right)=(1 / 2)\left|\rho\left(h_{1}\right)-\rho\left(h_{2}\right)\right|$ and multiplication by 2 gives

$$
\rho\left(h_{1}-h_{2}\right)=\left|\rho\left(h_{1}\right)-\rho\left(h_{2}\right)\right| .
$$

THEOREM 3.5. If $G$ is a space with constants, $\theta \neq h \in \bar{H} \cap U_{1}$, and $h_{0} \in \bar{H}$, there exists $\alpha \in R$ such that $\alpha h=h_{0}$. In particular if $\rho\left(h_{0}\right)<1$, then $h_{0}$ $= \pm\left(\rho\left(h_{0}\right) / \rho(h)\right) h$.

Proof. By Lemma 2.1, there exist $h_{0}^{\prime} \in U_{1-\rho(h)}$ and $\beta \in R$ such that $\beta h_{0}^{\prime}=h_{0}$. Now $h$ is a constant of $G$ and so either $\left\{h, h_{0}^{\prime}\right\}$ or $\left\{h,-h_{0}^{\prime}\right\}$ is positive. If $\left\{h, h_{0}^{\prime}\right\}$ is positive, then by Lemma $3.1,\left\{\left(\rho\left(h_{0}^{\prime}\right) / 2 \rho(h)\right) h,(1 / 2) h_{0}^{\prime}\right\}$ is positive. By Theorem 3.4, both these elements belong to $\bar{H}$ and so by Lemma 3.2,

$$
\begin{aligned}
\rho\left(\frac{\rho\left(h_{0}^{\prime}\right)}{2 \rho(h)} h-\frac{1}{2} h_{0}^{\prime}\right) & =\left|\rho\left(\frac{\rho\left(h_{0}^{\prime}\right)}{2 \rho(h)} h\right)-\rho\left(\frac{1}{2} h_{0}^{\prime}\right)\right| \\
& =\left|\frac{1}{2} \rho\left(h_{0}^{\prime}\right)-\frac{1}{2} \rho\left(h_{0}^{\prime}\right)\right|=0
\end{aligned}
$$

and so

$$
\frac{1}{2} h_{0}^{\prime}=\frac{\rho\left(h_{0}^{\prime}\right)}{2 \rho(h)} h \quad \text { and } \quad h_{0}=2 \beta\left(\frac{1}{2} h_{0}^{\prime}\right)=\frac{\beta \rho\left(h_{0}^{\prime}\right)}{\rho(h)} h .
$$

If $\left\{h,-h_{0}^{\prime}\right\}$ is positive we get $h_{0}=\left(-\beta \rho\left(h_{0}^{\prime}\right) / \rho(h)\right) h$ and the first part of the theorem is proved.

Now if $\rho\left(h_{0}\right)<1$, we may choose $h_{0}^{\prime}=(1-\rho(h)) h_{0}$ and $\beta=(1 /(1-\rho(h))$. Then $h_{0}= \pm\left(\rho\left(h_{0}\right) / \rho(h)\right) h$.

COROllary 1. If $G^{\prime}$ is a subspace of a space with constants, then $G^{\prime} \supset \bar{H}$.

Proof. Since $\theta \in G^{\prime}, H \cap U_{1} \subset G^{\prime}$ as $h \in H \cap U_{1}$ implies there exists an $n$ such that $n h=\theta$. Therefore $\alpha h \in G^{\prime}$ for all $\alpha \in R$ and so $\bar{H} \in G^{\prime}$. 
CoRollary 2. If $G^{\prime}$ is a closed subspace of a space with constants, then $G^{\prime}$ is a space with constants.

Proof. By Corollary 1, $G^{\prime} \supset \bar{H} \neq\{\theta\}$ and since it is closed it is complete.

LEMmA 3.3. If $G$ is a space with constants, and $\theta \neq h \in \bar{H} \cap U_{1}$, there exists a real number $\alpha_{h}>0$ such that $\alpha_{h} h=\theta$ and such that $0<\alpha<\alpha_{h}$ implies $\alpha h \neq \theta$.

Proof. Let $A=\{\alpha>0 \mid \alpha h=\theta\}$. By Lemma $2.2, A$ is equal to $\{\alpha \geqq 2 / \rho(h) \mid \alpha h$ $=\theta\}$ and by Lemma 2.3, $A$ is closed. Thus if $A$ is not empty, $\alpha_{h}=$ g.1.b. $\alpha \in A \alpha$ has the required property. But $A$ cannot be empty. For choose $h_{0} \in H$ such that $h_{0} \neq \theta$. Then there exist an in teger $n_{0}$ such that $n_{0} h_{0}=\theta$ and, by Theorem 3.5 , a real number $\alpha \neq 0$ such that $\alpha h=h_{0}$. Thus $\theta=n_{0}(\alpha h)=\left(n_{0} \alpha\right) h=-\left(n_{0} \alpha\right) h$ $=\left(-n_{0} \alpha\right) h$. Now either $n_{0} \alpha$ or $-n_{0} \alpha$ is positive and so belongs to $A$.

COROLLARY. $\alpha h=\theta$ if and only if $\alpha=n \alpha_{h}$ for some integer $n$.

Definition 3.4. Let $q_{h}=(1 / 2) \alpha_{h} \rho(h)$. By Lemma 2.2, $q_{h} \geqq 1$.

Lemma 3.4. If $G$ is a space with constants, and $h \in \bar{H} \cap U_{1}$ and $h \neq \theta$, then $\bar{H}$ is equivalent to $R_{2 q_{h}}$.

Proof. For $h_{0} \in \bar{H}$, there exists, by Theorem 3.5, $\alpha \in R$ such that $\alpha h=h_{0}$. Define $l_{h}: \bar{H} \rightarrow R_{2 q_{h}}$ by $l_{h}\left(h_{0}\right)=j(\alpha \rho(h))$. We show that $l_{h}$ is uniquely defined and gives an equivalence between $\bar{H}$ and $R_{2 q_{h}}$.

(a) If $h_{0}=\alpha h=\beta h$, then $(\beta-\alpha) h=\theta$ and $\beta-\alpha=n \alpha_{h}$ (corollary to Lemma 3.3). Thus $j(\alpha \rho(h))-j(\beta \rho(h))=j((\alpha-\beta) \rho(h))=j\left(n \alpha_{h} \rho(h)\right)=j\left(n\left(2 q_{h}\right)\right)=\theta$ and $l_{h}$ is uniquely defined.

(b) $l_{h}\left(h_{1}+h_{2}\right)=l_{h}\left(\alpha_{1} h+\alpha_{2} h\right)=j\left(\left(\alpha_{1}+\alpha_{2}\right) \rho(h)\right)=j\left(\alpha_{1} \rho(h)\right)+j\left(\alpha_{2} \rho(h)\right)=l_{h}\left(h_{1}\right)$ $+l_{h}\left(h_{2}\right)$ and $l_{h}$ is a homomorphism.

(c) If $l_{h}\left(h_{0}\right)=\theta$, then $\alpha \rho(h)=2 n q_{h}$ and $\alpha=n \alpha_{h}$ and $h_{0}=\alpha h=\theta$. Thus $l_{h}$ is an isomorphism.

(d) If $\rho\left(h_{0}\right)<1, h_{0}= \pm\left(\rho\left(h_{0}\right) / \rho(h)\right) h$ by Theorem 3.5. Thus $\left|j^{-1}\left(l_{h}\left(h_{0}\right)\right)\right|$ $=\left|j^{-1}\left(j\left(\left(\rho\left(h_{0}\right) / \rho(h)\right) \rho(h)\right)\right)\right|=\rho\left(h_{0}\right)$ and $l_{h}$ is an isometry on $\bar{H} \cap U_{1}$.

(e) Suppose $a \in U_{1} \subset R_{2 q_{h}}$. Let $\alpha=(1 / \rho(h))\left\{j^{-1}(a)\right\}$ and $h_{0}=\alpha h$. Then $h_{0} \in U_{1} \subset \bar{H}$ and $l_{h}\left(h_{0}\right)=j\left(j^{-1}(a)\right)=a$. Thus $l_{h}$ maps $U_{1} \cap \bar{H}$ onto $U_{1} \cap R_{2 q_{h}}$.

Thus (Definition 3.1) $l_{h}$ gives an equivalence between $\bar{H}$ and $R_{2 q_{h}}$.

CoRollaRy. If $h^{\prime} \in \bar{H} \cap U_{1}$ and $h^{\prime} \neq \theta$, then $q_{h^{\prime}}=q_{h}$ and $l_{h^{\prime}}= \pm l_{h}$.

Proof. By the lemma, $\bar{H}$ is equivalent to both $R_{\mathbf{2} q_{h}}$ and $R_{2 q_{h^{\prime}}}$ and so $R_{\mathbf{2} q_{h}}$ is equivalent to $R_{2 q_{h^{\prime}}}$. This implies immediately that $q_{h}=q_{h^{\prime}}$. Now the only continuous isomorphisms of $R_{2 q}$ onto itself are the identity and the reflection $(a \rightarrow-a)$. But $l_{h^{\prime}}\left(l_{h}^{-1}\right)$ is such a map and so $l_{h^{\prime}}= \pm l_{h}$.

Thus we may drop the subscript $h$ from $q_{h}$ and define $q=(1 / 2) \alpha_{h} \rho(h)$ for any $h \in \bar{H} \cap U_{1}$ such that $h \neq \theta$. We choose one of the two equivalence mappings of $\bar{H}$ onto $R_{2 q}$ and denote it by $l$. The other is then $-l$.

We have already proved 
THEOREM 3.6. If $G$ is a space with constants, then $l: \bar{H} \rightarrow R_{2 q}$ is a character of $\bar{H}$.

Theorem 3.7. If $G$ is a space with constants, then for each $a \in U_{1}$, there exists a character $L$ of $G$ such that $L=l$ on $\bar{H}$ and $\left|j^{-1}(L(a))\right|=\rho(a)$.

Proof. By Theorems 3.3, 3.4, and 3.6 there exist characters of $G$ equal to $l$ on $\bar{H}$. If $a \in \bar{H},\left|j^{-1}(L(a))\right|=\left|j^{-1}(l(a))\right|=\rho(a)$ and we are through. Suppose $a \notin \bar{H}$. For each $h \in \bar{H} \cap U_{1}, l(h)$ or $l(-h)=j(\rho(h))$. Choose $h_{0} \in \bar{H} \cap U_{1 / 2}$ such that $h_{0} \neq \theta$ and $l\left(h_{0}\right)=j\left(\rho\left(h_{0}\right)\right)$. Since $h_{0}$ is a constant of $G$, there exists $b$ such that $b= \pm(1 / 2) a$ and $\left\{h_{0}, b\right\}$ is positive. From the proof of Theorem 3.3, there is a character $L$ of $G$ equal to $l$ on $\bar{H}$ such that $L(b)=j(M)$ where $M$

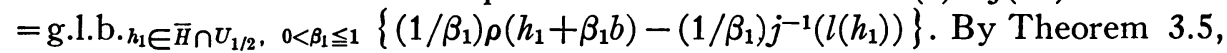
$h_{1}= \pm\left(\rho\left(h_{1}\right) / \rho\left(h_{0}\right)\right) h_{0}$.

(a) If $h_{1}=\left(\rho\left(h_{1}\right) / \rho\left(h_{0}\right)\right) h_{0}$, then by Lemma $3.1 \rho\left(h_{1}+\beta_{1} b\right)=\rho\left(h_{1}\right)+\beta_{1} \rho(b)$, and thus $\left(1 / \beta_{1}\right) \rho\left(h_{1}+\beta_{1} b\right)-\left(1 / \beta_{1}\right) j^{-1}\left(l\left(h_{1}\right)\right)=\left(1 / \beta_{1}\right) \rho\left(h_{1}\right)+\rho(b)-\left(1 / \beta_{1}\right) \rho\left(h_{1}\right)$ $=\rho(b)$.

(b) If $h_{1}=-\left(\rho\left(h_{1}\right) / \rho\left(h_{0}\right)\right) h_{0}$, then

$$
\frac{1}{\beta_{1}} \rho\left(h_{1}+\beta_{1} b\right)-\frac{1}{\beta_{1}} j^{-1}\left(l\left(h_{1}\right)\right) \geqq \rho(b)-\frac{1}{\beta_{1}} \rho\left(h_{1}\right)+\frac{1}{\beta_{1}} \rho\left(h_{1}\right)=\rho(b) .
$$

Thus $M=\rho(b)$ and $L(b)=j(\rho(b))$. Then

$$
\left|j^{-1}(L(a))\right|=\left|j^{-1}(L( \pm 2 b))\right|=\mid j^{-1}\left( \pm 2 L(b)|=2| j^{-1} j(\rho(b)) \mid=2 \rho(b)=\rho(a) .\right.
$$

Let $G$ be a space with constants. The set of characters of $G$ which are extensions of $l$ is a topological space under the point open topology. We denote this space by $S$.

TheOREM 3.8. The space $S$ is connected.

Proof. Suppose $L_{0}$ and $L_{1}$ belong to $S$. For $0 \leqq \alpha \leqq 1$ we define $L_{\alpha}: G \rightarrow R_{2 q}$ as follows. For $b \in G$, there exist $a \in U_{1}$ and $\gamma \in R$ such that $\gamma a=b$ (Lemma 2.1). We put $L_{\alpha}(b)=L_{0}(((1-\alpha) \gamma) a)+L_{1}((\alpha \gamma) a)$. Using strongly the fact that $L_{0}=L_{1}=l$ on $\bar{H}$, one may verify that $L_{\alpha}(b)$ is uniquely defined and that $L_{\alpha}$ $\in S$. Since $\left|j^{-1}\left(L_{\alpha}(b)-L_{\alpha_{0}}(b)\right)\right| \leqq\left|j^{-1} L_{0}\left(\left(\left(\alpha_{0}-\alpha\right) \gamma\right) a\right)\right|+\left|j^{-1} L_{1}\left(\left(\left(\alpha-\alpha_{0}\right) \gamma\right) a\right)\right|$ $\leqq 2|\gamma|\left|\alpha-\alpha_{0}\right|$, the map $\alpha \rightarrow L_{\alpha}$ is a continuous curve connecting $L_{0}$ to $L_{1}$ in $S$. Thus $S$ is connected.

TheOREM 3.9. The space $S$ is compact.

Proof. See Theorem 6.1 which is independently proved. A direct proof, duplicating the proof that the unit sphere in a conjugate space is compact in the weak-star topology, can be given.

TheOREM 3.10. If $\rho(a) \geqq 1$, there exists $L \in S$, such that $\left|j^{-1}(L(a))\right| \geqq 1$. 
Proof. Suppose $\left|j^{-1}(L(a))\right|<1$ for all $L \in S$. There exist $b \in U_{1 / 3}$ and $\beta \in R$ such that $\beta b=a$ (Lemma 2.1). Now $\rho(a) \geqq 1$ and so $|\beta| \geqq 3$ which implies that $\beta[L(b)-(1 / \beta) L(a)]=\beta L(b)-L(a)=\theta$ for all $L \in S$. The function $f: S \rightarrow R_{2 q}$ defined by $f(L)=L(b)-(1 / \beta) L(a)$ is continuous (Lemma 2.3 and the definition of the point open topology). Since $S$ is connected (Theorem 3.8), $f(S)$ is connected. Now the set $C=\left\{c \in R_{2 q} \mid \beta c=\theta\right\}$ is totally disconnected, and $f(S)<C$. Thus $f(S) \equiv c_{0}$. Moreover $\left|j^{-1}\left(c_{0}\right)\right| \leqq\left|j^{-1}(L(b))\right|$ $+\left|j^{-1}((1 / \beta) L(a))\right|<2 / 3$.

Let $h=l^{-1}\left(c_{0}\right)$. Then $L(b-h)=L(b)-c_{0}=(1 / \beta) L(a)$, and $\left|j^{-1}(L(b-h))\right|$ $<1 /|\beta|$ for all $L \in S$. But there exists $L_{0} \in S$ such that $\left|j^{-1}\left(L_{0}(b-h)\right)\right|=\rho(b-h)$ (Theorem 3.7) and so $\rho(b-h)<1 /|\beta|$. Thus $1>|\beta| \rho(b-h)=\rho(\beta b-\beta h)$ $=\rho(a-\beta h)$. But $l(\beta h)=\beta l(h)=\beta c_{0}=\theta$ and $\beta h=\theta$. But then $\rho(a)<1$, contradicting the hypothesis.

Theorems 3.7 and 3.10 combine to give

THEOREM 3.11. If $G$ is a space with constants and if $b \neq \theta$, then there exists $L \in S$ such that $L(b) \neq \theta$.

Theorem 3.12. A group $G$ is equivalent to a closed subspace $G^{\prime}$ of $R_{2 q}(X)$ for some $q \geqq 1$ and for some compact, connected space $X$, if and only if $G$ is a space with constants.

Proof. (a) By Theorem 2.3 and Corollary 2 to Theorem 3.5, $G^{\prime}$ is a space with constants. But $G$ is equivalent to $G^{\prime}$ and it follows that $G$ is a space with constants.

(b) Suppose $G$ is a space with constants. Then $G$ uniquely determines an $R_{2 q}$ for $q \geqq 1$ (Lemma 3.4 and corollary), and the space $S$ is a compact, connected space. We define $I(b)\{L\}=L(b)$ for all $L \in S$. The choice of the point open topology on $S$ insures that $I(b)$ is a continuous function on $S$ and so $I: G \rightarrow R_{2 q}^{S}$.

By $\mathrm{P}^{\prime} 1$ and Theorem 3.11, $I$ is an isomorphism. Since $U_{1}$ generates $G, I\left(U_{1}\right)$ generates $I(G)$. But $I$ is an isometry on $U_{1}$, by $\mathrm{P}^{\prime} 2$ and Theorem 3.7, and so $I\left(U_{1}\right) \subset R_{2 q}(S)$ and therefore $I(G) \subset R_{2 q}(S)$. Moreover if $\rho(I(b))<1$, then $\rho(b)<1$ (Theorem 3.10) and so $I\left(U_{1}\right)=\widehat{U}_{1} \subset I(G)$. Thus we have proved that $I: G \rightarrow I(G) \subset R_{2 q}(S)$ and that $G$ is equivalent to $I(G)$. It remains only to show that $I(G)$ is a closed subspace of $R_{2 q}(S)$.

Suppose $f \in I(G) \cap \widehat{U}_{1}$, then there exists $b \in U_{1}$ such that $I(b)=f$. Then $(\alpha f)(L)=\alpha(I(b)(L))=\alpha L(b)=L(\alpha b)=I(\alpha b)(L) \in I(G)$.

Now suppose that $f \in R_{2 q}(S), \rho(f)<1$ and for some $\alpha \neq 0, \alpha f \in I(G)$. Thus $\alpha f=I(b)$ for some $b \in G$. There exist $\beta \in R$ and $a \in U_{1}$ such that $\beta a=b$. Let $\gamma=\max [4,|4 \beta / \alpha|]$. Then $\gamma \alpha[(\beta / \gamma \alpha)(I(a)(L))-(1 / \gamma) f(L)]=\beta L(a)-\alpha f(L)$ $=L(b)-L(b)=\theta$. Then, as in the proof of Theorem 3.10, $(\beta / \gamma \alpha)(I(a)(L))$ $-(1 / \gamma) f(L) \equiv c_{0} \in R_{2 q}$ and $\rho\left(c_{0}\right)<1 / 2$. Let $h=l^{-1}\left(c_{0}\right)$. Then $(1 / \gamma) f=(\beta / \gamma \alpha) I(a)$ $-I(h) \in I(G) \cap \widehat{U}_{1}$ and so $\gamma((1 / \gamma) f)=f \in I(G)$. Thus $I(G)$ is a subspace of 
$R_{2 q}(S)$. Since an equivalence map is a local isometry in both directions, completeness is preserved and the completeness of $G$ implies that $I(G)$ is complete and therefore closed.

4. The associated Banach spaces. If $G$ is equivalent to $R_{2 q}(X)$, the elements $x$ of $X$ give rise to characters of $G$. We wish to be able to identify these characters in terms of the metric group properties of $G$. We begin by examining certain Banach spaces associated with $G$. In this section, $G$ is assumed to be a space with constants.

For $L_{0} \in S$, we denote by $G_{0}$ the set $\left\{a \in G \mid L_{0}(a)=\theta\right\}$.

Lemma 4.1. If $a \in G_{0}$, there exists $b \in G_{0} \cap U_{1}$ and $\beta \in R$ such that $\beta b=a$.

Proof. By Lemma 2.1, there exists $c \in U_{1 / 2}$ and $\beta \in R$ such that $\beta c=a$. Let $h=l^{-1}\left(L_{0}(c)\right)$. Then $L_{0}(c-h)=\theta$ and $\rho(c-h) \leqq \rho(c)+\rho(h)=\rho(c)+\left|j^{-1}\left(L_{0}(c)\right)\right|$ $\leqq 2 \rho(c)<1$. Thus $b=c-h \in G_{0} \cap U_{1}$. Then $\beta b=\beta(c-h)=\beta c-\beta h=a-\beta h$. But $l(\beta h)=L_{0}(\beta h)=L_{0}(\beta h-a)=-L_{0}(\beta(c-h))=-\beta L_{0}(c-h)=\theta$ and so $\beta h=\theta$ and $\beta b=a$.

Lemma 4.2. If $b_{1}, b_{2} \in G_{0} \cap U_{1}, \beta_{1}, \beta_{2} \in R$ and $\beta_{1} b_{1}=\beta_{2} b_{2}$, then $\left|\beta_{1}\right| \rho\left(b_{1}\right)$ $=\left|\beta_{2}\right| \rho\left(b_{2}\right)$ and for any $\alpha \in R,\left(\alpha \beta_{1}\right) b_{1}=\left(\alpha \beta_{2}\right) b_{2} \in G_{0}$.

Proof. From $\mathrm{P}^{\prime} 3,\left(\alpha \beta_{1}\right) b_{1}$ and $\left(\alpha \beta_{2}\right) b_{2} \in G_{0}$. Now if $\beta_{1}=\beta_{2}=0$, the conclusions follow immediately. Thus we may assume $\left|\beta_{1}\right| \geqq\left|\beta_{2}\right|$ and $\beta_{1} \neq 0$. Then $2 \beta_{1}\left((1 / 2) b_{1}-\left(\beta_{2} / 2 \beta_{1}\right) b_{2}\right)=\theta$ and so $(1 / 2) b_{1}-\left(\beta_{2} / 2 \beta_{1}\right) b \in \bar{H}$ (Theorem 3.4). But $l\left((1 / 2) b_{1}-\left(\beta_{2} / 2 \beta_{1}\right) b_{2}\right)=L_{0}\left((1 / 2) b_{1}-\left(\beta_{2} / 2 \beta_{1}\right) b_{2}\right)=\theta$ and so $(1 / 2) b_{1}$ $=\left(\beta_{2} / 2 \beta_{1}\right) b_{2}$ (Lemma 3.4). Therefore $(1 / 2) \rho\left(b_{1}\right)=\rho\left((1 / 2) b_{1}\right)=\rho\left(\left(\beta_{2} / 2 \beta\right) b_{2}\right)$ $=\left(\left|\beta_{2}\right| / 2\left|\beta_{1}\right|\right) \rho\left(b_{2}\right)$ and $\left|\beta_{1}\right| \rho\left(b_{1}\right)=\left|\beta_{2}\right| \rho\left(b_{2}\right)$. Moreover, multiplying our equality by $2 \alpha \beta_{1}$ gives $\left(\alpha \beta_{1}\right) b_{1}=\left(\alpha \beta_{2}\right) b_{2}$.

Using the $\beta$ and $b$ of Lemma 4.1 , we define

(1) for each $a \in G_{0}, \rho^{\prime}(a)=|\beta| \rho(b)$, and

(2) for each $a \in G_{0}$ and each $\alpha \in R, \alpha \times a=(\alpha \beta) b$.

The uniqueness of these definitions follows from Lemma 4.2.

Let $G_{0}^{\prime}$ be the space whose elements and underlying algebraic group structure are those of $G_{0}$, but with this new metric and multiplication by reals. That is, using $a^{\prime}$ to denote the element $a$ in $G_{0}^{\prime}$, we have $\left\|a^{\prime}\right\|=\rho^{\prime}(a)$ and $\alpha a^{\prime}$ $=(\alpha \times a)^{\prime}$.

One may readily verify

Theorem 4.1. Go is a Banach space, and $G_{0}^{\prime}$ is equivalent to $G_{0}$.

Let $G^{\prime}$ be the vector direct sum of $G_{0}^{\prime}$ and the reals, $G^{\prime}=G_{0}^{\prime} \oplus R e$. For $a^{\prime}+\alpha e \in G^{\prime}$, we define $\left\|a^{\prime}+\alpha e\right\|=\gamma \rho(((1 / \gamma) \times a)+h)$ where $\gamma>\max \left[2\left\|a^{\prime}\right\|\right.$, $2|\alpha|]$ and $h=l^{-1}(j(\alpha / \gamma))$.

Lemma 4.3. $\left\|a^{\prime}+\alpha e\right\|$ is uniquely defined.

Proof. Suppose $\gamma_{1}, \gamma_{2}>\max \left[2\left\|a^{\prime}\right\|, 2|\alpha|\right]$ and $\gamma_{1} \geqq \gamma_{2}$. Then $l\left(\left(\gamma_{2} / \gamma_{1}\right) h_{2}\right)$ 
$=\left(\gamma_{2} / \gamma_{1}\right) l\left(h_{2}\right)=\left(\gamma_{2} / \gamma_{1}\right) j\left(\alpha / \gamma_{2}\right)=j\left(\alpha / \gamma_{1}\right)=l\left(h_{1}\right)$ and $\left(\gamma_{2} / \gamma_{1}\right) h_{2}=h_{1}$. Thus $\left(\gamma_{2} / \gamma_{1}\right)$ $\cdot\left(\left(1 / \gamma_{2}\right) \times a+h_{2}\right)=\left(\gamma_{2} / \gamma_{1}\right)\left(\left(\beta / \gamma_{2}\right) b+h_{2}\right)=\left(\beta / \gamma_{1}\right) b+h_{1}=\left(1 / \gamma_{1}\right) \times a+h_{1}$. Thus $\gamma_{1} \rho\left(\left(1 / \gamma_{1}\right) \times a+h_{1}\right)=\gamma_{1} \rho\left(\left(\gamma_{2} / \gamma_{1}\right)\left(\left(1 / \gamma_{2}\right) \times a+h_{2}\right)\right)=\gamma_{2} \rho\left(\left(1 / \gamma_{2}\right) \times a+h_{2}\right)$.

A direct verification then gives

TheOREM 4.2. $G^{\prime}=G_{0}^{\prime} \oplus$ Re is a Banach space.

Definition 4.1. An element $b$ of a Banach space $B$ is a unit element if for every $a \in B$, either $\|a+b\|=\|a\|+1$ or $\|a-b\|=\|a\|+1$ [8].

Lemma 4.4. The element $e=\theta^{\prime}+1 e \in G^{\prime}$ is a unit element.

Proof. For any $a^{\prime}+\alpha e \in G^{\prime}$, choose $\gamma>\max \left[2\left\|a^{\prime}\right\|, 2|\alpha|+2\right]$. Now $\bar{h}$ $=l^{-1}(j(1 / \gamma))$ is a constant of $G$. Assume $\{(1 / \gamma) \times a+h, \bar{h}\}$ is positive. Then $\left\|a^{\prime}+\alpha e+e\right\|=\gamma \rho\left((1 / \gamma) \times a+l^{-1}(j((\alpha+1) / \gamma))\right)=\gamma \rho((1 / \gamma) \times a+h+\bar{h})$ $=\gamma \rho((1 / \gamma) \times a+h)+\gamma \rho(\bar{h})=\left\|a^{\prime}+\alpha e\right\|+1$. If $\{-(1 / \gamma) \times a-h, \bar{h}\}$ is positive, the same argument gives $\left\|a^{\prime}+\alpha e-e\right\|=\left\|a^{\prime}+\alpha e\right\|+1$.

Lemma 4.5. $\lambda_{0}: G^{\prime} \rightarrow R$, defined by $\lambda_{0}\left(a^{\prime}+\alpha e\right)=\alpha$, is a linear functional of norm 1.

Proof. $\lambda_{0}$ is clearly linear and clearly $\left\|\lambda_{0}\right\| \geqq 1$. But $\left|\lambda_{0}\left(a^{\prime}+\alpha e\right)\right|=|\alpha|$ $=|\gamma||\alpha / \gamma|=|\gamma| \mid j^{-1}\left(L_{0}((1 / \gamma) \times a+h)|\leqq| \gamma \mid \rho((1 / \gamma) \times a+h)=\left\|a^{\prime}+\alpha e\right\|\right.$ and so $\left\|\lambda_{0}\right\|=1$.

For a fixed $q \geqq 1$, there is a natural mapping of $C(X)$, the Banach space of bounded, continuous, real-valued functions on $X$, into $R_{2 q}(X)$ given by $(j(b))(x)=j(b(x))$. For $G^{\prime}(X) \subset C(X)$, we assume for $j\left(G^{\prime}(X)\right)$ the metric, group properties induced on it as a subset of $R_{2 q}(X)$.

Theorem 4.3. If $X$ is compact, then $j(C(X))$ is equivalent to $R_{2 q}(X)$.

Proof. Theorem 1 of [4].

Lemma 4.6. If $X$ is connected, and if $G^{\prime}(X)$ is a linear subspace of $C(X)$ containing the function $e(x) \equiv 1$, then $j\left(G^{\prime}(X)\right)$ is a subspace of $R_{2 q}(X)$.

Proof. Since the map $j$ is a homomorphism, $j\left(G^{\prime}(X)\right)$ is an algebraic subgroup of $R_{2 q}(X)$.

(a) Suppose $a^{\prime}(x) \in G^{\prime}(X)$ and $j\left(a^{\prime}(x)\right) \in U_{1} \subset R_{2 q}(X)$. Consider the function $j^{-1}\left(j\left(a^{\prime}(x)\right)\right)-a^{\prime}(x)=f(x)$. Since $j^{-1}$ is continuous on $U_{1}, f$ is continuous and since $X$ is connected, $f(X)$ is a connected set. But $j(f(x)) \equiv \theta$ and so $f(X)$ $C I_{2 q}=\{n(2 q)\}$. Thus $j^{-1}\left(j\left(a^{\prime}(x)\right)\right)=a^{\prime}(x)+2 n_{0} q e(x)$ for some fixed integer $n_{0}$. Thus $j^{-1}\left(j\left(a^{\prime}(x)\right)\right)$ and $\alpha j^{-1}\left(j\left(a^{\prime}(x)\right)\right) \in G^{\prime}(X)$. Then $\alpha\left\{j\left(a^{\prime}(x)\right)\right\}=j\left(\alpha j^{-1}\left(j\left(a^{\prime}(x)\right)\right)\right)$ $\in j\left(G^{\prime}(X)\right)$.

(b) Now suppose $b(x) \in U_{1} \subset R_{2 q}(X)$ and for some $\alpha \neq 0, \alpha b(x)=j\left(a^{\prime}(x)\right)$ for some $a^{\prime}(x) \in G^{\prime}(X)$. Choose $\gamma>\max \left((4 /|\alpha|)\left\|a^{\prime}(x)\right\|\right.$, 4). Then $\gamma \alpha\left[(1 / \gamma) b(x)-j\left(a^{\prime}(x) / \gamma \alpha\right)\right] \equiv \theta$. Then by Theorem 3.4 and Lemma 2.4 $(1 / \gamma) b(x)-j\left(a^{\prime}(x) / \gamma \alpha\right) \equiv j(\beta e(x)) \in j\left(G^{\prime}(X)\right)$. Thus $(1 / \gamma) b(x) \in j\left(G^{\prime}(X)\right) \cap U_{1}$. 
By (a), $\gamma((1 / \gamma) b(x))=b(x) \in j\left(G^{\prime}(X)\right)$.

THEOREM 4.4. If $X$ is connected and $G^{\prime}(X)$ is a linear subspace of $C(X)$, then $G$ is equivalent to $j\left(G^{\prime}(X)\right)$ if and only if

(1) $G^{\prime}(X)$ contains $e(x) \equiv 1$, and

(2) there exists an equivalence map of $G^{\prime}=G_{0}^{\prime} \oplus$ Re onto $G^{\prime}(X)$ such that $e \rightarrow \pm e(x)$ under this equivalence.

Proof. (a) Suppose $G$ is equivalent to $j\left(G^{\prime}(X)\right)$. Let $i: G \rightarrow j\left(G^{\prime}(X)\right)$ be the equivalence map. Then for $\theta \neq h \in \bar{H} \subset G, i(h) \in \bar{H} \subset R_{2 q}(X)$ and by Lemma 2.4, $i(h)=j(\beta e(x))$ for some $\beta \neq 2 n q$ for any $n$. Thus, there exists $f(x) \in G^{\prime}(X)$ such that $j(f(x))=j(\beta e(x))$. Since $X$ is connected, $f(x)=\left(\beta+2 n_{0} q\right) e(x)$ and since $G^{\prime}(X)$ is a linear subspace and $\beta+2 n_{0} q \neq 0, e(x) \in G^{\prime}(X)$. Thus condition (1) is satisfied.

We proceed to prove condition (2). It is clear that the map $i(h)=j(\beta)$ is an equivalence map of $\bar{H}$ onto $R_{2 q}$. Thus on $\bar{H}, i= \pm l$. Define $\bar{e}(x)=+e(x)$ or $\bar{e}(x)=-e(x)$ depending on whether $i=+l$ or $i=-l$. Then for $a^{\prime}+\alpha e \in G^{\prime}$, choose $\gamma>\left\|a^{\prime}\right\|$ and define $I\left(a^{\prime}+\alpha e\right)=\gamma\left\{j^{-1}[i((1 / \gamma) \times a)]\right\}+\alpha \bar{e}(x)$.

(1) $I$ is uniquely defined for if $\delta \geqq \gamma,(1 / \delta) \times a=(\gamma / \delta)((1 / \gamma) \times a)$ and

$$
\begin{aligned}
\delta\left\{j^{-1}\left[i\left(\frac{1}{\delta} \times a\right)\right]\right\} & =\delta\left\{j^{-1}\left[i\left(\frac{\gamma}{\delta}\left(\frac{1}{\gamma} \times a\right)\right)\right]\right\} \\
& =\delta\left\{j^{-1}\left[\frac{\gamma}{\delta} i\left(\frac{1}{\gamma} \times a\right)\right]\right\} \\
& =\delta\left\{j^{-1} j\left[\frac{\gamma}{\delta}\left(j^{-1}\left(i\left(\frac{1}{\gamma} \times a\right)\right)\right)\right]\right\} \\
& =\gamma\left\{j^{-1}\left[i\left(\frac{1}{\gamma} \times a\right)\right]\right\} .
\end{aligned}
$$

(2) $I\left(G^{\prime}\right) \subset G^{\prime}(X)$. For if $a^{\prime}+\alpha e \in G^{\prime}, i((1 / \gamma) \times a)=j(f(x))$ for some $f(x)$ in $G^{\prime}(X)$. Then $\gamma\left\{j^{-1}[i((1 / \gamma) \times a)]\right\}+\alpha \bar{e}(x)=\gamma(f(x)+2 n q e(x))+\alpha \bar{e}(x) \in G^{\prime}(X)$.

(3) $I(e)=\bar{e}(x)$.

(4) $I$ is linear. It is clearly a homomorphism. Moreover if $b^{\prime}=\beta a^{\prime}=(\beta \times a)^{\prime}$, $I\left(b^{\prime}+\beta(\alpha e)\right)=\gamma|\beta|\left\{j^{-1}[i((1 / \gamma|\beta|) \times b)]\right\}+\beta \alpha \bar{e}(x)=\gamma|\beta|\left\{j^{-1}[i((1 / \gamma|\beta|)\right.$ $\times(\beta \times a))]\}+\beta \alpha \bar{e}(x)=\gamma|\beta|(\beta /|\beta|)\left\{j^{-1}[i((1 / \gamma) \times a)]\right\}+\beta \alpha \bar{e}(x)=\beta I\left(a^{\prime}+\alpha e\right)$.

(5) $I$ is norm-preserving. For if $\left\|a^{\prime}+\alpha e\right\|<1 / 6,|\alpha|<1 / 6$ by Lemma 4.5 and so $\left\|a^{\prime}\right\|<1 / 3$ by the triangle inequality. Then we may put $\gamma=1$ in the definition of $\left\|a^{\prime}+\alpha e\right\|$, and we have

$$
\begin{aligned}
\left\|a^{\prime}+\alpha e\right\| & =\rho(a+h)=\rho(i(a+h))=\rho(i(a)+i(h)) \\
& =\rho\left(i(a)+j\left\{\left(j^{-1} l(h)\right)(\bar{e}(x))\right\}\right)=\left\|j^{-1}\left(i(a)+j\left\{\left(j^{-1} l(h)\right)(\bar{e}(x))\right\}\right)\right\| \\
& =\left\|j^{-1}(i(a)+j(\alpha \bar{e}(x)))\right\|=\left\|j^{-1}(i(a))+\alpha \bar{e}(x)\right\|=\left\|I\left(a^{\prime}+\alpha e\right)\right\|
\end{aligned}
$$


(again taking $\gamma=1$ ). Thus $I$ is norm-preserving on $U_{1 / 6}$. But $I$ is linear and so $I$ is norm-preserving on $G^{\prime}$.

(6) $I$ maps $G^{\prime}$ onto $G^{\prime}(X)$. For suppose $b^{\prime}(x) \in G^{\prime}(X)$. There exists $a \in G_{0}$ and $h \in \bar{H}$ such that $i(a+h)=j\left(b^{\prime}(x)\right)$. But $j\left(I\left(a^{\prime}\right)\right)=i(a)=j\left(b^{\prime}(x)\right)-i(h)$ $=j\left(b^{\prime}(x)\right)-j\left\{\left(j^{-1} l(h)\right)(\bar{e}(x))\right\}=j\left\{b^{\prime}(x)-\left(j^{-1} l(h)\right)(\bar{e}(x))\right\}$ and $I\left(a^{\prime}\right)=b^{\prime}(x)$ $-\left(j^{-1} l(h)+2 n q\right)(\bar{e}(x))$. Thus $I\left(a^{\prime}+\left(j^{-1} l(h)+2 n q\right) e\right)=b^{\prime}(x)$.

Thus $I$ is a Banach space equivalence and clearly an equivalence in our sense.

(b) Now suppose $I: G^{\prime} \rightarrow G^{\prime}(X)$ is the hypothesized equivalence. Since $l^{-1} L_{0}: G \rightarrow \bar{H}$ is a continuous projection, $G=G_{0} \oplus \bar{H}$ is a direct sum. Thus we may define $J: G \rightarrow j\left(G^{\prime}(X)\right)$ by $J(a+h)=j\left\{I\left(a^{\prime}\right)+\left(j^{-1}(l(h))\right) I(e)\right\}$.

(1) $J$ is clearly a homomorphism.

(2) If $J(a+h)=\theta, \quad j\left\{I\left(a^{\prime}\right)+\left(j^{-1}(l(h))\right) I(e)\right\} \equiv \theta \quad$ and $\quad I\left(a^{\prime}\right) \equiv[2 n q$ $\left.+j^{-1}(l(h))\right] I(e)$ (since $\left.I(e)=\bar{e}(x)\right)$. But $G^{\prime}$ and therefore $G^{\prime}(X)$ is a direct sum and so $I\left(a^{\prime}\right) \equiv 0$ and $j^{-1}(l(h))=-2 n q=0$ as $-q<j^{-1}(l(h)) \leqq q$. Thus $a=h=\theta$ and $J$ is an isomorphism.

(3) If $f(x) \in j\left(G^{\prime}(X)\right)$, there exist $a \in G_{0}$ and $\alpha \in R$ such that $f(x)=j\left(I\left(a^{\prime}\right)\right.$ $+\alpha I(e))=j\left\{I\left(a^{\prime}\right)+\left(j^{-1}(j(\alpha))\right) I(e)\right\}$. Let $h=l^{-1}(j(\alpha))$; then $J(a+h)=f(x)$ and $J$ maps $G$ onto $j\left(G^{\prime}(X)\right)$.

(4) Suppose $\rho(a+h)<1$. Now $\rho(J(a+h))=\left\|j^{-1}\left\{j\left(I\left(a^{\prime}\right)+\left(j^{-1}(l(h))\right) I(e)\right)\right\}\right\|$. Since $j^{-1} j(\alpha)=\alpha$ for $|\alpha|<1$, we prove $\rho(J(a+h))=\rho(a+h)$ by showing that $\left\|I\left(a^{\prime}\right)+\left(j^{-1}(l(h))\right) I(e)\right\|=\rho(a+h)<1$. Now $G^{\prime}$ and $G^{\prime}(X)$ are Banach spaces and so an equivalence between them in our sense is a Banach space equivalence. Thus $\left\|I\left(a^{\prime}\right)+\left(j^{-1}(l(h))\right) I(e)\right\|=\left\|a^{\prime}+j^{-1}(l(h)) e\right\|$. Now $\rho(h)=\left|j^{-1}(l(h))\right|$ $=\left|j^{-1}\left(L_{0}(h)\right)\right|=\left|j^{-1}\left(L_{0}(a+h)\right)\right| \leqq \rho(a+h)<1$. Thus choosing $\gamma=\max [4$, $\left.4\left\|a^{\prime}\right\|\right]$, we have $\left\|a^{\prime}+j^{-1}(l(h)) e\right\|=\gamma \rho((1 / \gamma) \times a+(1 / \gamma) h)$ since $(1 / \gamma) h$ $=l^{-1}\left\{j\left[(1 / \gamma) j^{-1}(l(h))\right]\right\}$. But $\gamma\{(1 / \gamma) \times a+(1 / \gamma) h-(1 / \gamma)(a+h)\}=\theta$ and so $b=(1 / \gamma) \times a+(1 / \gamma) h-(1 / \gamma)(a+h) \in \bar{H}$. But $l(b)=L_{0}(b)=\theta$ and so $b=\theta$. Therefore $\gamma \rho((1 / \gamma) \times a+(1 / \gamma) h)=\gamma \rho((1 / \gamma)(a+h))=\rho(a+h)$. Thus $J$ is an isometry on $U_{1} \subset G$.

(5) Suppose $\rho(J(a+h))<1$. Then for some fixed integer $n_{0}, 2 n_{0} q-1$ $<I\left(a^{\prime}\right)+j^{-1}(l(h)) I(e)<2 n_{0} q+1$ for all $x \in X$. Thus $\| I\left(a^{\prime}\right)+\left\{j^{-1}(l(h))-2 n_{0} q\right\}$ $\cdot I(e)\|=\| a^{\prime}+\left\{j^{-1}(l(h))-2 n_{0} q\right\} e \|<1$. By Lemma 4.5, $\left|j^{-1}(l(h))-2 n_{0} q\right|<1$ and since $-q<j^{-1}(l(h)) \leqq q$ we have $n_{0}=0$ and $\rho(h)=\left|j^{-1}(l(h))\right|<1$. Thus again $\gamma \rho((1 / \gamma) \times a+(1 / \gamma) h)=\rho(J(a+h))<1$. But since $\gamma \rho((1 / \gamma) \times a+(1 / \gamma) h)$ $<1, \gamma \rho((1 / \gamma) \times a+(1 / \gamma) h)=\rho(a+h)$ and $J$ maps $U_{1} \subset G$ onto $U_{1} \subset j\left(G^{\prime}(X)\right)$.

Thus $G$ is equivalent to $j\left(G^{\prime}(X)\right)$.

5. Some theorems on Banach spaces. We have shown that a group $G$ is equivalent to $R_{2 q}(X)$ for some compact, connected space $X$ if and only if (1) $G$ is a space with constants and (2) $G^{\prime}$ is equivalent to $C(X)$ (Theorems 4.3 and 4.4). In the usual characterizations of a Banach space $G^{\prime}$ as $C(X)$, the points of $X$ are found in $G^{\prime *}$ (the set of linear functionals of $G^{\prime}$ ). We wish to give a characterization in terms of the group $G$. In $\S 6$, we show that the characters 
of $G$ correspond naturally to a subset of the linear functionals of $G_{0}^{\prime}$. However, the $F_{T}$ 's of $G_{0}^{\prime}$ [8], or the extreme points of the unit sphere of $G_{0}^{\prime *}$ [2], do not in general give the required space $X$.

Specifically, for $G^{\prime}=G_{0}^{\prime} \oplus R e$, we look for a space $E \subset G_{0}^{\prime *}$ such that the natural correspondence $a^{\prime}+\alpha e \rightarrow \xi\left(a^{\prime}\right)+\alpha(\xi \in E)$ is an equivalence, and such that $G^{\prime}$ is equivalent to $C(X)$ for some $X$ if and only if this mapping takes $G^{\prime}$ on to $C(E)$.

Let $B^{\prime}$ be a Banach space with a unit element $e$.

Definition 5.1. If $\lambda_{0}$ is a linear functional on $B^{\prime}$ of norm 1 whose value at $e$ is 1 , then $B=\left\{b \in B^{\prime} \mid \lambda_{0}(b)=0\right\}$ is a positive hyperplane of $B^{\prime} . B$ clearly is a Banach space and $B^{\prime}=B \oplus R e$ is a direct sum.

Definition 5.2. A functional $\lambda \in B^{*}$ is essentially positive (relative to $B^{\prime}$ ) if for all $b \in B$, and $\alpha \in R,|\lambda(b)+\alpha| \leqq\|b+\alpha e\|$.

In what follows the topology in $B^{*}$ is the weak-star (point open) topology.

LEMma 5.1. The set $\mathcal{S}$ of essentially positive linear functionals is closed in $B^{*}$.

Proof. Suppose $\lambda^{\prime} \in B^{*}$ and $\lambda^{\prime} \in \mathcal{S}$. Then there exists $b \in B, \alpha \in R$ such that $\left|\lambda^{\prime}(b)+\alpha\right|>\|b+\alpha e\|$. The set $V=\left\{\lambda \in B^{*}|| \lambda(b)-\lambda^{\prime}(b) \mid<\left(\left|\lambda^{\prime}(b)+\alpha\right|\right.\right.$ $-\|b+\alpha e\|) / 2\}$ is open and contains $\lambda^{\prime}$. For $\lambda \in V,|\lambda(b)+\alpha| \geqq\left|\lambda^{\prime}(b)+\alpha\right|$ $-\left|\lambda(b)-\lambda^{\prime}(b)\right|>\left(\left|\lambda^{\prime}(b)+\alpha\right|+\|b+\alpha e\|\right) / 2>\|b+\alpha e\|$. Thus $V \cap S=\phi$ and $\mathcal{S}$ is closed.

Lemma 5.2. $\delta$ is compact.

Proof. For $\alpha=0, \lambda \in \mathcal{S},|\lambda(b)| \leqq\|b\|$ for all $b \in B$. Thus $S$ is contained in $\Sigma$, the unit sphere in $B^{*}$. But $\Sigma$ is compact in the weak-star topology [1], $\delta$ is closed by Lemma 5.1 , and $S$ is compact.

Definition 5.3. For $\lambda \in \mathcal{S}, M(\lambda)=\left\{b \in B \mid \lambda(b) \geqq \lambda^{\prime}(b)\right.$ for all $\left.\lambda^{\prime} \in \mathcal{S}\right\}$.

We may order the sets $M(\lambda)$ by inclusion.

Definition 5.4. A functional $\xi \in \mathcal{S}$ is a maximal functional of $\mathcal{S}$ if $M(\xi)$ is a maximal set in the ordering of the sets $M(\lambda)$.

It can be shown that in the natural imbedding of $\delta$ into $\Sigma^{\prime}$ (the unit sphere in $B^{\prime *}$ ), the maximal functionals do not in general map into either $F_{T}$ 's or extreme points of $\Sigma^{\prime}$.

Theorem 5.1. If $\lambda_{0} \in \mathcal{S}$, there exists a maximal functional $\xi$, such that $M(\xi) \supset M\left(\lambda_{0}\right)$.

Proof. By Zorn's lemma, $M\left(\lambda_{0}\right)$ is contained in a maximal linearly ordered chain $\left\{M\left(\lambda_{\mu}\right)\right\}$. Define $E(\mu)=\left\{\lambda \in \mathcal{S} \mid \lambda(b)=\lambda_{\mu}(b)\right.$ for all $\left.b \in M\left(\lambda_{\mu}\right)\right\}$.

(1) $E(\mu)$ is not empty as $\lambda_{\mu} \in E(\mu)$.

(2) If $M\left(\lambda_{\mu_{1}}\right) \subset M\left(\lambda_{\mu_{2}}\right)$ then $E\left(\mu_{1}\right) \supset E\left(\mu_{2}\right)$. For if $\lambda \in E\left(\mu_{2}\right), \lambda(b)=\lambda_{\mu_{2}}(b)$ $\geqq \lambda_{\mu_{1}}(b)$ for all $b \in M\left(\lambda_{\mu_{2}}\right)$. Thus $\lambda(b) \geqq \lambda_{\mu_{1}}(b)$ for all $b \in M\left(\lambda_{\mu_{1}}\right)$. But the opposite inequality always holds and so $\lambda(b)=\lambda_{\mu_{1}}(b)$ for all $b \in M\left(\lambda_{\mu_{1}}\right)$ and so $\lambda \in E\left(\mu_{1}\right)$. 
(3) $E(\mu)$ is closed for $E(\mu)=\bigcap_{b \in M\left(\lambda_{\mu}\right)}\left\{\lambda \in \mathcal{S} \mid \lambda(b)=\lambda_{\mu}(b)\right\}$ and is the intersection of closed sets.

Thus $\{E(\mu)\}$ is a family of closed, non-empty sets of $\mathcal{S}$, linearly ordered by inclusion. Since $S$ is compact, there exists $\xi \in \bigcap_{\mu}\{E(\mu)\}$. For any $\lambda_{\mu}$ in our chain, we now have $\xi(b)=\lambda_{\mu}(b) \geqq \lambda(b)$ for all $b \in M\left(\lambda_{\mu}\right)$ and all $\lambda \in S$. Thus $M(\xi) \supset M\left(\lambda_{\mu}\right)$. If $M\left(\lambda_{\mu^{\prime}}\right) \supset M(\xi)$, then $M\left(\lambda_{\mu^{\prime}}\right)$ belongs to the chain (the chain is maximal) and so $M(\xi) \supset M\left(\lambda_{\mu^{\prime}}\right)$. Thus $\xi$ is a maximal functional and since $M\left(\lambda_{0}\right) \in\left\{M\left(\lambda_{\mu}\right)\right\}, M(\xi) \supset M\left(\lambda_{0}\right)$.

TheOREM 5.2. If $B$ is a positive hyperplane of $B^{\prime}$, a Banach space with a unit element $e$, then for each $b^{\prime} \in B^{\prime}, b^{\prime}=b+\beta e$, there exists a maximal functional $\xi$ of $S$ such that $|\xi(b)+\beta|=\left\|b^{\prime}\right\|$.

Proof. By the Hahn-Banach extension theorem $[3$, p. 28], there exists a $\lambda_{0}^{\prime} \in B^{\prime *}$ such that $\left\|\lambda_{0}^{\prime}\right\|=1, \lambda_{0}^{\prime}(e)=1$, and $\lambda_{0}^{\prime}\left(b^{\prime}\right)=\inf _{\alpha \in R}\left\|b^{\prime}+\alpha e\right\|-\alpha$. Now $e$ is a unit element. We assume first that $\left\|b^{\prime}+e\right\|=\left\|b^{\prime}\right\|+1$. Then for $\alpha \geqq 0$, Lemma 3.1 implies that $\left\|b^{\prime}+\alpha e\right\|-\alpha=\left\|b^{\prime}\right\|+\alpha-\alpha=\left\|b^{\prime}\right\|$. (The condition $\left\|b^{\prime}\right\|+\alpha\|e\|<1$ is not needed in a Banach space.) For $\alpha<0,\left\|b^{\prime}+\alpha e\right\|-\alpha$ $\geqq\left\|b^{\prime}\right\|-|\alpha|-\alpha=\left\|b^{\prime}\right\|$. Thus $\inf _{\alpha \in R}\left\|b^{\prime}+\alpha e\right\|-\alpha=\left\|b^{\prime}\right\|$ and $\lambda_{0}^{\prime}\left(b^{\prime}\right)=\left\|b^{\prime}\right\|$.

Let $\lambda_{0}$ be the functional $\lambda_{0}^{\prime}$ cut down to $B$. Since $\left\|\lambda_{0}^{\prime}\right\|=1$ and $\lambda_{0}^{\prime}(e)=1, \lambda_{0}$ is an element of $\mathcal{S}$. Moreover for all $\lambda \in \mathcal{S}, \lambda(b)+\beta \leqq\|b+\beta e\|$ and so, for the $b$ and $\beta$ defined by $b^{\prime}, \lambda(b) \leqq\|b+\beta e\|-\beta=\lambda_{0}(b)$. Thus $b \in M\left(\lambda_{0}\right)$. But there exists a maximal functional $\xi$ such that $M(\xi) \supset M\left(\lambda_{0}\right)$, Theorem 5.1. Moreover on $M\left(\lambda_{0}\right), \xi=\lambda_{0}$ and so $\xi(b)+\beta=\lambda_{0}(b)+\beta=\lambda_{0}^{\prime}(b+\beta e)=\lambda_{0}^{\prime}\left(b^{\prime}\right)=\left\|b^{\prime}\right\|$.

Now if $\left\|b^{\prime}-e\right\|=\left\|-b^{\prime}+e\right\|=\left\|b^{\prime}\right\|+1$, the same argument proves the existence of a maximal functional $\xi$, such that $\xi(-b)-\beta=\left\|b^{\prime}\right\|$. Since one of these two conditions must apply we have shown the existence of a maximal functional $\xi$, such that $|\xi(b)+\beta|=\left\|b^{\prime}\right\|$.

THEOREM 5.3. If $B$ is a positive hyperplane of $B^{\prime}$, a Banach space with a unit element $e$, and $E$ is the space of maximal functionals of $S$, then $B^{\prime}$ is equivalent to a closed, linear subspace of $C(E)$.

Proof. We map $b^{\prime}=b+\beta e \rightarrow f(\xi)=\xi(b)+\beta$. The weak star topology on $E \subset B^{*}$ insures the continuity of $f$. Since $|\xi(b)+\beta| \leqq\|b+\beta e\|, f(\xi)$ is bounded. This map of $B^{\prime} \rightarrow C(E)$ is clearly linear, and by Theorem 5.2 it is norm-preserving. Thus $B^{\prime}$ is equivalent to its image in $C(E)$ and since $B^{\prime}$ is a complete, linear space, its image is a closed, linear subspace of $C(E)$.

TheOREM 5.4. A Banach space $B^{\prime}$ is equivalent to $C(X)$ for some compact space $X$ if and only if

(1) $B^{\prime}$ has a unit element and

(2) there exists a positive hyperplane $B$ of $B^{\prime}$, such that for any $b \in B$ and $\beta \in R$, there exists $\bar{b} \in B$ and $\bar{\beta} \in R$, such that $\xi(\bar{b})+\bar{\beta}=|\xi(b)+\beta|$ for all maximal functionals $\xi \in S$. 
Proof. (a) Suppose $B^{\prime}$ is equivalent to $C(X)$ for some compact $X$. Then $e(x) \equiv 1$ is a unit element. Let $B$ be any positive hyperplane of $B^{\prime}$ (one exists by the Hahn-Banach theorem). To show that condition (2) is necessary we need only show that every maximal functional corresponds to a point of $X\left(\xi(b)=b\left(x_{0}\right)\right.$ for some $\left.x_{0} \in X\right)$, for $b^{\prime}(x) \in C(X)$ implies $\left|b^{\prime}(x)\right| \in C(X)$.

Let $X_{b}=\left\{x \in X \mid b(x)=\sup _{x \in X} b(x)\right\}$. Since $X$ is compact $X_{b}$ is not empty. Now the functional $\lambda_{0}: b \rightarrow b\left(x_{0}\right)$ is an element of $\mathcal{S}$. Thus for any $\lambda \in \mathcal{S}$ and $b \in M(\lambda), \quad \lambda(b) \geqq \lambda_{0}(b)=b\left(x_{0}\right)$ for all $x_{0} \in X$. Thus for $b \in M(\lambda), \quad \lambda(b)$ $\geqq \sup _{x \in x} b(x)$. Now choose $\alpha=\|b\|$. Then $\lambda(b) \leqq\|b+\alpha e\|-\alpha=\sup _{x \in X}(b(x)$ $+\alpha e(x))-\alpha=\sup _{x \in X} b(x)$. Thus $\lambda(b)=\sup _{x \in X} b(x)$ for $b \in M(\lambda)$. Now for $b_{i}$ any finite set of elements of $M(\lambda)$ we have $\sum_{i=1}^{n} b_{i} \in M(\lambda)$ and so

$$
\sup _{x \in X}\left[\sum_{i=1}^{n} b_{i}(x)\right]=\lambda\left(\sum_{i=1}^{n} b_{i}\right)=\sum_{i=1}^{n}\left(\lambda\left(b_{i}\right)\right)=\sum_{i=1}^{n} \sup _{x \in X}\left(b_{i}(x)\right) .
$$

But this implies that $\bigcap_{i=1}^{n} X_{b_{i}}$ is not empty. Since $X$ is compact and $X_{b}$ is closed we have that there exists an $x_{1} \in X$ such that $x_{1} \in \bigcap_{b \in M(\lambda)} X_{b}$. Then $\lambda_{1}: b \rightarrow b\left(x_{1}\right)$ is equal to $\lambda$ on $M(\lambda)$ and so we have $M\left(\lambda_{1}\right) \supset M(\lambda)$. Now suppose $\lambda=\xi$ is a maximal functional. Thus $M(\xi)=M\left(\lambda_{1}\right)$ and $\xi(b)=b\left(x_{1}\right)$ for all $b \in M\left(\lambda_{1}\right)$. But $b \in B$ such that there exists an $\alpha \in R$ such that $b\left(x_{1}\right)+\alpha$ $=\|b+\alpha e\|$ certainly belong to $M\left(\lambda_{1}\right)$. Thus $\xi=\lambda_{1}$ on these elements and by Lemma 2.3 of [8], $\xi=\lambda_{1}$ on $B$ and so all maximal functionals correspond to points of $X$. \{The preceding also proves that all points of $X$ give rise to maximal functionals. $\}$

(b) Now suppose (1) and (2) are satisfied. Let $\bar{E}$ be the closure of $E$ in $B^{*}$. Since $E \subset \mathcal{S}$, and $\mathcal{S}$ is compact, $\bar{E}$ is compact. Moreover, the map $b^{\prime}=b$ $+\alpha e \rightarrow f(\xi)=\xi(b)+\alpha$ for $\xi \in \bar{E}$ is an equivalence map (Theorem 5.3, the addition of elements of $S$ to $E$ to form $\bar{E}$ does not change this property). Thus $B$ is equivalent to $\Gamma$, a closed, linear subspace of $C(\bar{E})$. Then by the theorem of Kakutani [6], $\Gamma=C(\bar{E})$ if

(1) whenever $\xi_{1}, \xi_{2} \in \bar{E}$ and $\xi_{1} \neq \xi_{2}$ there exists $f \in \Gamma$ such that $f\left(\xi_{1}\right) \neq f\left(\xi_{2}\right)$,

(2) $\Gamma$ contains a nontrivial constant function, and

(3) $\Gamma$ is lattice closed. $\xi_{1}=\xi_{2}$.

If $f\left(\xi_{1}\right)=f\left(\xi_{2}\right)$ for all $f$ in $\Gamma$, then $\xi_{1}(b)+\alpha=\xi_{2}(b)+\alpha$ for all $b \in B$, and so

Moreover $0+e \in B^{\prime}$ maps into the function $f(\xi) \equiv 1$ and $\Gamma$ contains a nontrivial constant function.

Finally

$$
\begin{aligned}
\max _{\min }\left\{\xi\left(b_{1}\right)+\alpha_{1}, \xi\left(b_{2}\right)+\alpha_{2}\right\} & \\
= & \frac{1}{2}\left\{\xi\left(b_{1}\right)+\xi\left(b_{2}\right)+\alpha_{1}+\alpha_{2} \pm\left|\xi\left(b_{2}\right)-\xi\left(b_{1}\right)+\alpha_{2}-\alpha_{1}\right|\right\}
\end{aligned}
$$

and by condition (2) both these functions are in $\Gamma$, and $\Gamma$ is lattice closed. 
Thus $B^{\prime}$ is equivalent to $C(\bar{E})$. By the remark in the proof of the converse all the elements of $\bar{E}$ are maximal and so $\bar{E}=E$.

Lemma 5.3. If $X$ is compact, then $X$ is connected if and only if $e(x) \equiv 1$ and $e(x) \equiv-1$ are the only unit elements of $C(X)$.

Proof. (a) If $V$ is a nontrivial open and closed set in $X$, then $e(x) \equiv 1$ on $V$ and $e(x) \equiv-1$ on the complement of $V$ is a unit element of $C(X)$.

(b) Suppose $f \in C(X)$ is a unit element. Then $\|f\|=\|0+f\|=\|0\|+1=1$, and so $|f(x)| \leqq 1$ for all $x$. Now suppose that for some $x_{0} \in X,\left|f\left(x_{0}\right)\right|<1$. There exists $b \in C(X)$ such that $\|b\|=1, b\left(x_{0}\right)=1$, and $b(x) \equiv 0$ wherever $f(x)=1$. Then $\|b+f\|<2$ which contradicts the hypothesis that $f$ is a unit element. Thus if $f$ is a unit element, $|f(x)| \equiv 1$ for all $x \in X$. But $X$ is connected and so either $f(x) \equiv 1$ or $f(x) \equiv-1$.

Suppose $B^{\prime}$ is a Banach space with a unit element $e$, and $B_{1}$ and $B_{2}$ are positive hyperplanes of $B^{\prime}$. For $b_{2} \in B_{2}$, there is a unique $b_{1} \in B_{1}$ and $\alpha \in R$ such that $b_{2}=b_{1}+\alpha e$. For $\lambda_{1} \in \mathcal{S}_{1}$ we define $\left[i\left(\lambda_{1}\right)\right]\left(b_{2}\right)=\lambda_{1}\left(b_{1}\right)+\alpha$. It is clear that $i$ is a $1-1$ map of $S_{1}$ onto $S_{2}$.

Lemma 5.4. If $\xi_{1} \in \mathcal{S}_{1}$ is a maximal functional of $\mathcal{S}_{1}$, then $i\left(\xi_{1}\right) \in \mathcal{S}_{2}$ is a maximal functional of $\boldsymbol{S}_{2}$.

Proof. Let $M_{2}=\left(M\left(\xi_{1}\right)+R e\right) \cap B_{2}$. For $b_{2} \in M_{2}, \quad\left[i\left(\xi_{1}\right)\right]\left(b_{2}\right)=\xi_{1}\left(b_{1}\right)+\alpha$ $\geqq \lambda_{1}\left(b_{1}\right)+\alpha=\left[i\left(\lambda_{1}\right)\right]\left(b_{2}\right)$ for all $\lambda_{1} \in \mathcal{S}_{1}$. Thus $M\left\{i\left(\xi_{1}\right)\right\} \supset M_{2}$. Suppose $M\left(\lambda_{2}\right)$ $\supset M\left(i\left(\xi_{1}\right)\right)$, and $M\left(\lambda_{2}\right) \neq M\left(i\left(\xi_{1}\right)\right)$. Then $M_{1}=\left(M\left(\lambda_{2}\right)+R e\right) \cap B_{1}$ contains $M\left(\xi_{1}\right)$ properly and moreover $M\left(i^{-1}\left(\lambda_{2}\right)\right) \supset M_{1}$. But this contradicts the maximality of $\xi_{1}$ and so $M\left(\lambda_{2}\right)=M\left(i\left(\xi_{1}\right)\right)$ and $i\left(\xi_{1}\right)$ is a maximal functional of $\delta_{2}$.

6. A characterization of $R_{2 q}(X)$. Let $G$ be a space with constants. For $L \in S$, we define the functional $I_{0}(L): G_{0}^{\prime} \rightarrow R$ by $\left[I_{0}(L)\right]\left(a^{\prime}\right)=\alpha j^{-1}(L((1 / \alpha)$ $\times a)$ ) for $|\alpha|>\left\|a^{\prime}\right\|$. The notation is that of $\S \S 3$ and 4 .

Lemma 6.1. $I_{0}(L)$ is uniquely defined.

Proof. The ambiguity of definition arises in the choice of $\alpha$. However, for $|\gamma| \geqq|\alpha|,(\alpha / \gamma)((1 / \alpha) \times a)=(\alpha / \gamma) \times((1 / \alpha) \times a)=(1 / \gamma) \times a$. Thus $\gamma j^{-1}(L((1 / \gamma) \times a))=\gamma j^{-1}(L((\alpha / \gamma)((1 / \alpha) \times a)))=\gamma j^{-1}((\alpha / \gamma) L((1 / \alpha) \times a))=\gamma j^{-1}$ $\left(j\left((\alpha / \gamma) j^{-1} L((1 / \alpha) \times a)\right)\right)=\gamma\left((\alpha / \gamma) j^{-1}(L((1 / \alpha) \times a))\right)=\alpha j^{-1}(L((1 / \alpha) \times a))$.

Lemma 6.2. The functional $I_{0}(L)$ is an element of $S_{0}$, the set of positive linear functionals of $G_{0}^{\prime}$ (with respect to $G^{\prime}$ ).

Proof. (1) $\left[I_{0}(L)\right]\left(a_{1}^{\prime}+a_{2}^{\prime}\right)=\alpha j^{-1}\left(L\left((1 / \alpha) \times\left(a_{1}+a_{2}\right)\right)\right)=\alpha j^{-1}\left(L\left((1 / \alpha) \times a_{1}\right)\right.$ $\left.+L\left((1 / \alpha) \times a_{2}\right)\right)$. But we may choose $\alpha>\left\|a_{1}^{\prime}\right\|+\left\|a_{2}^{\prime}\right\|$ which makes $j^{-1}$ a homomorphism and so $I_{0}(L)$ is a homomorphism.

(2) $\left[I_{0}(L)\right]\left((\beta \times a)^{\prime}\right)=\alpha j^{-1} L((1 / \alpha) \times(\beta \times a))$ where we may choose $\alpha$ $>\max \left[|\beta|\left\|a^{\prime}\right\|,\left\|a^{\prime}\right\|\right]$. Then $\alpha j^{-1} L((1 / \alpha) \times(\beta \times a))=\alpha j^{-1} L(\beta \times((1 / \alpha) \times a))$ $=\alpha j^{-1} L(\beta((1 / \alpha) \times a))=\alpha j^{-1} \beta L((1 / \alpha) \times a)=\alpha j^{-1}\left(j\left(\beta j^{-1}(L((1 / \alpha) \times a))\right)\right)=\beta\left(\alpha j^{-1}\right.$ $L((1 / \alpha) \times a))=\beta\left[I_{0}(L)\right]\left(a^{\prime}\right)$ and so $I_{0}(L)$ is linear. 
(3) For $a^{\prime}+\beta e \in G^{\prime}$, choose $\alpha>\left\|a^{\prime}\right\|+|\beta|$ and put $h=l^{-1}(j(\beta / \alpha))$. Then $\left|\left[I_{0}(L)\right]\left(a^{\prime}\right)+\beta\right|=\left|\alpha j^{-1}(L((1 / \alpha) \times a))+\beta\right|=\alpha\left|j^{-1}(L((1 / \alpha) \times a))+\beta / \alpha\right|$ $=\alpha\left|j^{-1}(L((1 / \alpha) \times a)+j(\beta / \alpha))\right|=\alpha\left|j^{-1}(L((1 / \alpha) \times a+h))\right| \leqq \alpha \rho((1 / \alpha) \times a+h)$ $=\left\|a^{\prime}+\beta e\right\|$ and so $I_{0}(L) \in \mathcal{S}_{0}$.

Lemma 6.3. The map $I_{0}: S \rightarrow \mathcal{S}_{0}$ is a homeomorphism onto.

Proof. (1) If $I_{0}\left(L_{1}\right)=I_{0}\left(L_{2}\right)$, then $L_{1}=L_{2}$ on $G_{0} \cap U_{1}$ and since $G_{0} \cap U_{1}$ generates $G_{0}, L_{1}=L_{2}$ on $G_{0}$. But $L_{1}=L_{2}=l$ on $\bar{H}$ and so $L_{1}=L_{2}$ on $G$. Thus $L_{1}=L_{2}$ and $I_{0}$ is $1-1$.

(2) Suppose $\lambda \in \mathcal{S}_{0}$. Define $\bar{L}: G \rightarrow R_{2 q}$ by $\bar{L}(a+h)=j\left(\lambda\left(a^{\prime}\right)\right)+l(h) . \bar{L}$ is certainly a homomorphism and so satisfies $\mathrm{P}^{\prime} 1$. Moreover, if $\rho(a+h)<1$, $\rho(h)<1$ and $\rho(a+h)=\left\|a^{\prime}+\left(j^{-1}(l(h))\right) e\right\|$. (See proof of Theorem 4.4.) Then $1>\rho(a+h) \geqq\left|\lambda\left(a^{\prime}\right)+j^{-1}(l(h))\right|=\left|j^{-1}\left(j\left(\lambda\left(a^{\prime}\right)+j^{-1}(l(h))\right)\right)\right|=\left|j^{-1}(\bar{L}(a+h))\right|$ and so $\bar{L}$ satisfies $\mathrm{P}^{\prime} 2$. Thus by Theorem $3.2, \bar{L}$ is a character of $G$. Since $\bar{L}=l$ on $\bar{H}$, by definition, we have $\bar{L} \in S$. But $\left[I_{0}(\bar{L})\right]\left(a^{\prime}\right)=\alpha j^{-1} \bar{L}((1 / \alpha) \times a)$ $=\alpha j^{-1}\left(j \lambda\left(((1 / \alpha) \times a)^{\prime}\right)\right)=\alpha \lambda\left(((1 / \alpha) \times a)^{\prime}\right)=\lambda\left(a^{\prime}\right)$ and so $I_{0}(\bar{L})=\lambda$ and $I_{0}$ maps $S$ on to $S_{0}$.

(3) Since $S_{0}$ is compact (Lemma 5.2), and $S$ is clearly Hausdorff, to show $I_{0}$ is a homeomorphism we need only show that $I_{0}^{-1}$ is continuous. Now for $\bar{\lambda} \in \mathcal{S}_{0}, a_{i} \in G_{0}, i=1, \cdots, n$, and $1 \geqq \epsilon>0, V=\left\{L \in S|| j^{-1}\left(L\left(a_{i}\right)-\left[I_{0}^{-1}(\bar{\lambda})\right]\left(a_{i}\right)\right) \mid\right.$ $<\epsilon\}$ is a basic neighborhood of $I_{0}^{-1}(\bar{\lambda})$ in $S$. We need choose the $a_{i}$ 's only from $G_{0}$ as for all $L \in S, L=l$ on $\bar{H}$ and $G=G_{0} \oplus \bar{H}$. Let $V^{\prime}=\left\{\lambda \in \mathcal{S}_{0}|| \lambda\left(a_{i}^{\prime}\right)\right.$ $\left.-\bar{\lambda}\left(a_{i}^{\prime}\right) \mid<\epsilon\right\}$. Thus $V^{\prime}$ is a neighborhood of $\bar{\lambda}$ in $S_{0}$. If $L \in I_{0}^{-1}\left(V^{\prime}\right)$ we have that

$$
\begin{aligned}
\left|j^{-1}\left(L\left(a_{i}\right)-\left[I_{0}^{-1}(\bar{\lambda})\right]\left(a_{i}\right)\right)\right| & =\left|j^{-1}\left(L\left(\alpha_{i}\left(\left(1 / \alpha_{i}\right) \times a_{i}\right)\right)-\left[I_{0}^{-1}(\bar{\lambda})\right]\left(\alpha_{i}\left(\left(1 / \alpha_{i}\right) \times a_{i}\right)\right)\right)\right| \\
& =\left|j^{-1}\left(\alpha_{i}\left(L\left(\left(1 / \alpha_{i}\right) \times a_{i}\right)-\alpha_{i}\left[I_{0}^{-1}(\bar{\lambda})\right]\left(\left(1 / \alpha_{i}\right) \times a_{i}\right)\right)\right)\right| \\
& =\mid j^{-1} j\left(\alpha_{i} j^{-1} L\left(\left(1 / \alpha_{i}\right) \times a_{i}\right)\right. \\
& \left.\quad-\alpha_{i}\left[I_{0}^{-1}(\bar{\lambda})\right]\left(\left(1 / \alpha_{i}\right) \times a_{i}\right)\right) \mid \\
& =\left|j^{-1} j\left(\left[I_{0}(L)\right]\left(a_{i}^{\prime}\right)-\bar{\lambda}\left(a_{i}^{\prime}\right)\right)\right| \\
& =\left|\left[I_{0}(L)\right]\left(a_{i}^{\prime}\right)-\bar{\lambda}\left(a_{i}^{\prime}\right)\right|<\epsilon,
\end{aligned}
$$

since $j^{-1} j(\beta)=\beta$ if $|\beta|<1$. Thus $I_{0}^{-1}\left(V^{\prime}\right) \subset V, I_{0}^{-1}$ is continuous and $I_{0}$ is a homeomorphism.

We have immediately

THEOREM 6.1. If $G$ is a space with constants, $S$ is compact.

Definition 6.1. For $\bar{L} \in S$ and $L_{0} \in S$, let $N_{0}(\bar{L})=\left\{a \in G_{0} \cap U_{1} \mid j^{-1}(\bar{L}(a))\right.$ $\geqq j^{-1}(L(a))$ for all $\left.L \in S\right\}$.

As in $\$ 5$ we order the sets $N_{0}(\bar{L})$ by inclusion.

Definition 6.2. $F \in S$ is a maximal $G_{0}$ character if $N_{0}(F)$ is a maximal set in the ordering. 
The correspondence between $N_{0}(\bar{L})$ and $M\left(I_{0}(\bar{L})\right)$ \{Definition 5.3\} is quite direct.

Lemma 6.4. $M\left(I_{0}(\bar{L})\right)=\left\{a^{\prime} \in G_{0}^{\prime} \mid\right.$ for $\left.\alpha>\left\|a^{\prime}\right\|,(1 / \alpha) \times a \in N_{0}(\bar{L})\right\}$.

Proof. $\left(I_{0}(\bar{L})\right)\left(a^{\prime}\right)=\alpha j^{-1}(\bar{L}((1 / \alpha) \times a))$. Since $\alpha>0,\left(I_{0}(\bar{L})\right)\left(a^{\prime}\right) \geqq\left(I_{0}(L)\right)\left(a^{\prime}\right)$ $\rightarrow j^{-1}(\bar{L}((1 / \alpha) \times a)) \geqq j^{-1}(L((1 / \alpha) \times a))$ and the lemma follows as $I_{0}$ maps $S$ onto $S_{0}$ (Lemma 6.3).

A corollary of Lemma 6.4, obtained by putting $\alpha=1$, is

Lemma 6.5. $N_{0}(\bar{L})=\left\{a \in G_{0} \mid a^{\prime} \in M\left(I_{0}(\bar{L})\right)\right.$ and $\left.\left\|a^{\prime}\right\|<1\right\}$.

THEOREM 6.2. $F$ is a maximal $G_{0}$ character if and only if $I_{0}(F)$ is a maximal functional of $S_{0}$.

Proof. (a) Suppose $M(\lambda) \supset M\left(I_{0}(F)\right)$; then, by Lemma 6.5, $N_{0}\left(I_{0}^{-1}(\lambda)\right)$ $\supset N_{0}(F)$. But then if $F$ is maximal, $N_{0}\left(I_{0}^{-1}(\lambda)\right) \subset N_{0}(F)$ and, by Lemma 6.4, $M(\lambda) \subset M\left(I_{0}(F)\right)$. Thus $M\left(I_{0}(F)\right)$ is maximal and $I_{0}(F)$ is a maximal functional of $S_{0}$.

(b) Suppose $N_{0}(L) \supset N_{0}(F)$. Then Lemma 6.4 implies $M\left(I_{0}(L)\right)$ $\supset M\left(I_{0}(F)\right)$. But if $I_{0}(F)$ is maximal, $M\left(I_{0}(L)\right) \subset M\left(I_{0}(F)\right)$ and by Lemma 6.5, $N_{0}(L) \subset N_{0}(F)$. Thus $N_{0}(F)$ is maximal and $F$ is a maximal $G_{0}$ character.

The maximality of $F \in S$ does not depend on the choice of $L_{0}$.

Definition 6.3. $F \in S$ is a maximal character if it is a maximal $G_{0}$ character for all $L_{0} \in S$.

THEOREM 6.3. $F$ is a maximal character if it is a maximal $G_{0}$ character for some $L_{0} \in S$.

Proof. The theorem is an immediate consequence of Theorem 6.2 and Lemma 5.4.

The final characterization may now be given.

TheOREM 6.4. A group $G$ is equivalent to $R_{2 q}(X)$ for some $q \geqq 1$ and for some compact, connected space $X$ if and only if

(1) there exists a unique, isomorphic, isometry, $i_{a}:[0, \rho(a)] \rightarrow G$ such that $i_{a}(\rho(a))=a$,

(2) the elements of $\bar{H} \cap U_{1}$ are constants of $G$,

(3) the elements of $\bar{H} \cap U_{1}$ are the only constants of $G$,

(4) for $b \in G$, there exists $b \in G$ such that $j^{-1}\{F(\bar{b})\}=\left|j^{-1}\{F(b)\}\right|$ for all maximal characters $F$ of $S$.

Proof. Suppose $G$ is equivalent to $R_{2 q}(X)$ for $q \geqq 1$ and for $X$ a compact, connected space. Conditions (1) and (2) follow from Theorems 2.1, 2.2, and 2.3. Condition (3) follows quickly since a constant of $R_{2 q}(X)$ must be of the form $f(x) \equiv a \in U_{1} \subset R_{2 q}$ (see proof of Lemma 5.3) and so $f \in \bar{H} \cap U_{1}$. Now by 
Theorems 4.3 and $4.4, G^{\prime}$ is equivalent to $C(X)$. Then by Theorem 5.4, there exists a positive hyperplane $\tilde{G}$ of $G^{\prime}$ such that $G^{\prime}=\tilde{G} \oplus R e$ and such that for each $a^{\prime} \in \widetilde{G}$ and each $\alpha \in R$, there exists $\bar{a}^{\prime} \in \widetilde{G}$ and $\bar{\alpha} \in R$ such that for all maximal functionals $\xi$ of $\delta, \xi\left(\bar{a}^{\prime}\right)+\bar{\alpha}=\left|\xi\left(a^{\prime}\right)+\alpha\right|$. Let $\lambda_{0}$ be the functional which defined $\tilde{G}$. We redefine $G_{0}$ using the character $L_{0}=I_{0}^{-1}\left(\lambda_{0}\right)$. Then $G=G_{0} \oplus \bar{H}$ and $G_{0}^{\prime}=\widetilde{G}$. Now suppose $b \in U_{1}$. We have $b=a+h$ where $a \in G_{0}$ and $h \in \bar{H}$. Then there exist $\bar{a}^{\prime} \in G_{0}^{\prime}$ and $\bar{\alpha} \in R$ such that $\xi\left(\bar{a}^{\prime}\right)+\bar{\alpha}=\mid \xi\left(a^{\prime}\right)$ $+j^{-1}(l(h)) \mid$, for all maximal functionals $\xi$. Let $\bar{b}=\bar{a}+l^{-1}(j(\bar{\alpha}))$. Now by Theorems 6.2 and 6.3 , the maximal characters $F$ are exactly the elements $I_{0}^{-1}(\xi)$ where $\xi$ are the maximal functionals. Thus $j^{-1}\{F(\bar{b})\}=j^{-1}\left\{\left(I_{0}^{-1}(\xi)\right)(\bar{b})\right\}$ $=j^{-1}\left\{j\left(\xi\left(\bar{a}^{\prime}\right)\right)+j(\bar{\alpha})\right\}=j^{-1} j\left\{\xi\left(\bar{a}^{\prime}\right)+\bar{\alpha}\right\}=j^{-1} j\left|\xi\left(a^{\prime}\right)+j^{-1}(l(h))\right|=\mid j^{-1} j\left(\xi\left(a^{\prime}\right)\right.$ $\left.+j^{-1}(l(h))\right)|=| j^{-1}(F(b)) \mid$ and condition (4) is satisfied.

(b) If (1) and (2) are satisfied, $G$ is a space with constants. Choosing any $L_{0} \in S$, we have, as before, $G=G_{0} \oplus \bar{H}, G^{\prime}=G_{0}^{\prime} \oplus R e, e$ is a unit element of $G^{\prime}$, and $G_{0}^{\prime}$ is a positive hyperplane of $G^{\prime}$. For each $a^{\prime} \in G_{0}^{\prime}$, and each $\alpha \in R$, choose $\gamma>4 \max \left(\left\|a^{\prime}\right\|,|\alpha|\right)$ and let $h=l^{-1} j(\alpha / \gamma)$ and $b=(1 / \gamma) \times a+h$. By condition (4), there exists $\bar{b}=\bar{c}+\bar{h} \in G$, such that $j^{-1}(F(\bar{b}))=\left|j^{-1}(F(b))\right|$ for all maximal characters $F$. Let $\bar{a}^{\prime}=(\gamma \times \bar{c})^{\prime}$ and $\bar{\alpha}=\gamma j^{-1}(l(\bar{h}))$. Then for $\xi$ a maximal functional of $S_{0}, \xi\left(\bar{a}^{\prime}\right)+\bar{\alpha}=\left(I_{0}(F)\right)\left(\bar{a}^{\prime}\right)+\bar{\alpha}=\gamma j^{-1}(F((1 / \gamma) \times(\gamma \times \bar{c})))$ $+\gamma j^{-1}(l(\bar{h}))=\gamma j^{-1} F(\bar{b})=\left|\gamma j^{-1}(F(b))\right|=\left|\gamma j^{-1} F((1 / \gamma) \times a)+\gamma j^{-1} l(h)\right|=\mid \xi\left(a^{\prime}\right)$ $+\alpha \mid$. Thus by Theorem $5.4, G^{\prime}$ is equivalent to $C(X)$ for some compact $X$. Moreover if $a^{\prime}+\alpha e$ is a unit of $G^{\prime}$, then $(1 / \gamma) \times a+h$ is a constant of $G$. By condition (3), $(1 / \gamma) \times a+h \in \bar{H}$ and so $a^{\prime}=\theta$. But then $|\alpha|$ must equal 1 and so $\pm e$ are the only unit elements of $G^{\prime}$. But $e(x) \equiv 1$ and $e(x) \equiv-1$ are unit elements of $C(X)$ and so $e$ must map into either $e(x) \equiv 1$ or $e(x) \equiv-1$ under the equivalence and these are the only unit elements of $C(X)$ and so $X$ is connected (Lemma 5.3). Finally by Theorems 4.4 and $4.3, G$ is equivalent to $R_{2 q}(X)$.

\section{The homeomorphism theorem.}

TheOREM 7.1. If $X$ and $Y$ are compact, and if $R_{2 q_{1}}(X)$ is equivalent to $R_{2 q_{2}}(Y)$, then $q_{1}=q_{2}$ and $X$ is homeomorphic to $Y$.

Proof. Let $T: R_{2 q_{1}}(X) \rightarrow R_{2 q_{2}}(Y)$ be the equivalence. Choose a positive integer $n$ such that $2 q_{1} / n<1$. Then $f_{1} \equiv j\left(2 q_{1} / n\right) \in R_{2 q_{1}}(X), n f_{1}=\theta$ and $\rho\left(f_{1}\right)$ $=2 q_{1} / n<1$. Thus $n\left(T f_{1}\right)=\theta$, and $\rho\left(T f_{1}\right)=2 q_{1} / n$. Since $Y$ is compact, there exists $y_{0} \in Y$ such that $j^{-1}\left(\left(T f_{1}\right)\left(y_{0}\right)\right)= \pm 2 q_{1} / n$. But $n\left(\left(T f_{1}\right)\left(y_{0}\right)\right)=\theta$ so that $n j^{-1}\left(\left(T f_{1}\right)\left(y_{0}\right)\right)=2 m q_{2}$, where $m$ is an integer. Thus $q_{1}= \pm m q_{2}$ and $q_{1}$ is an integer multiple of $q_{2}$. The exact same proof, using $T^{-1}$, gives that $q_{2}$ is an integer multiple of $q_{1}$. Since $q_{1}$ and $q_{2}$ are both positive we have that $q_{1}=q_{2}$.

Now define the mapping $T^{*}: C(X) \rightarrow C(Y)$ by

$$
\left(T^{*} \sigma\right)(y)=\gamma\left[j^{-1}\{T(j((1 / \gamma) \sigma))(y)\}\right]
$$

for $\gamma>\|\sigma\|$. It is easy to verify that $T^{*}$ is a uniquely defined, linear, norm- 
preserving map of $C(X)$ onto $C(Y)$. By the Banach-Stone theorem [10], $X$ is homeomorphic to $Y$.

\section{BIBLIOGRAPHY}

1. L. Alaoglu, Weak topologies of normed linear spaces, Ann. of Math. vol. 41 (1940) pp. 252-267.

2. R. F. Arens and J. L. Kelly, Characterizations of the space of continuous functions over a compact Hausdorff space, Trans. Amer. Math. Soc. vol. 62 (1947) pp. 499-508.

3. S. Banach, Théorie des opérations linéaires, Warsaw, 1932.

4. S. Eilenberg, Sur les transformations d'espaces métriques en circonference, Fund. Math. vol. 24 (1935) pp. 160-176.

5. M. Jerison, The space of bounded maps into a Banach space, Ann. of Math. vol. 52 (1950) pp. 309-327.

6. S. Kakutani, Concrete representation of abstract (M) spaces, Ann. of Math. vol. 42 (1941) pp. 994-1024.

7. I. Kaplansky, Topological rings, Bull. Amer. Math. Soc. vol. 54 (1948) pp. 809-826.

8. S. B. Myers, Banach spaces of continuous functions, Ann. of Math. vol. 49 (1948) pp. 132-140.

9. - Normed linear spaces of continuous functions, Bull. Amer. Math. Soc. vol. 56 (1950) pp. 233-241.

10. M. H. Stone, Applications of the theory of Boolean rings to general topology, Trans. Amer. Math. Soc. vol. 41 (1937) pp. 375-481.

\section{UNIVERSITY OF MICHIGAN, ANN ARbor, Mich. \\ WAYNE UNIVERSITY, Detroit, Mich.}

